J o u r n a l of

Mathematics

and Applications

JMA No 42, pp 147-185 (2019)

\title{
A Minimax Approach to Mapping Partial Interval Uncertainties into Point Estimates
}

\author{
Vadim Romanuke
}

\begin{abstract}
A problem of simultaneously reducing a group of interval uncertainties is considered. The intervals are positively normalized. There is a constraint, by which the sum of any point estimates taken from those intervals is equal to 1 . Hence, the last interval is suspended. For mapping the interval uncertainties into point estimates, a minimax decision-making method is suggested. The last interval's point estimate is then tacitly found. Minimax is applied to a maximal disbalance between a real unknown amount and a guessed amount. These amounts are interpreted as aftermaths of the point estimation. According to this model, the decision-maker is granted a pure strategy, whose components are the most appropriate point estimates. Such strategy is always single. Its components are always less than the right endpoints. The best mapping case is when we obtain a totally regular strategy whose components are greater than the left endpoints. The irregular strategy's components admitting many left endpoints are computed by special formulae. The worst strategy exists, whose single component is greater than the corresponding left endpoint. Apart from the point estimation, irregularities in the decision-maker's optimal strategy may serve as an evidence of the intervals' incorrectness. The irregularity of higher ranks is a criterion for correcting the intervals.
\end{abstract}

AMS Subject Classification: 91A05, 91A35, 90C47.

Keywords and Phrases: Game theory; Interval uncertainties; Minimax decisionmaking; Pure strategy; Point estimates.

COPYRIGHT (c) by Publishing House of Rzeszów University of Technology P.O. Box 85, 35-959 Rzeszów, Poland 


\section{Introduction}

Interval estimates are unavoidable in modeling processes whose parameters are generated with a variety of stochastic or weakly controllable factors. It is a poor practice to deal with such estimates because conclusions and inferences from them come "intervally" uncertain (e.g., [4, 13, 49, 19]). For reducing interval uncertainties, both expert judgments and statistically observed data are used $[1,21,23]$. Ideally, this is Bayesian decision making. Practically, a faithful elicitation of the probability and utility functions is almost always prohibitive $[23,18,46]$. Estimations based on expert judgments allow narrowing the interval in a limited number of steps $[41,50]$. When no expert or other statistical data are available, decisions on reducing intervals are made with special policies/strategies $[4,47,20,2]$.

On the other hand, point estimates are much less reliable. As the interval becomes shorter, reliability of the interval estimate requires more statistical data $[9,36]$. For performing a decision analysis involving fuzzy logic, we need to substantiate a membership function and a method of defuzzification $[22,43]$. Their substantiation also relies on statistical observations. In general, a proper/valid mapping of the interval estimate into a point estimate takes huge statistics $[17,47,46]$. Is the mapping possible without statistical data? Can we "guess" the most appropriate point value that could substitute the interval? These questions are answered positively only by using a conception of the least risk under the worst conditions that could happen. In other words, this is about minimax principle.

In decision making, minimax principle allows forgetting the absence of any statistics. In contrast to decisions using expected value or expected utility, being nonprobabilistic is a key feature of minimax decision making. Minimax robustness is used in statistical decision theory, where a deterministic parameter is estimated under uncertainty $[3,35]$. Minimax is also used in designing linear estimators, where solutions of convex optimization problems give the optimal, minimax regret-minimizing linear estimator $[32,6,17]$. The main ground for trusting the use of minimax principle is that it minimizes losses $[42,26]$. Minimaxing risks/damages is a common routine in studying processes with highly volatile and non-controllable parameters $[4,19,14,3]$. Then game models are involved. Their solutions may propose both pure and mixed strategies $[44,33]$. Pure strategies are quite acceptable for a decision-maker, whereas mixed ones contain probabilities (or probabilistic measures). Practical realization of probabilities is inseparably associated with relative statistical frequencies. The frequencies tend to the corresponding probabilities by some statistical data $[46,39,9$, 11]. But if we have those data, we can map the interval estimate into a point estimate without minimaxing. Then no game modeling is needed anymore.

Availability of statistical data excludes reasonability of the minimax mixed strategies. Therefore, only pure strategies are reasonable to make non-statistical decisions on point estimates. This task becomes severer for multiple interval uncertainties representing a group of connected/intertwined parameters $[13,19,34,12,8]$. Indeed, if intervals are closed, then we have to map a multidimensional hyperparallelepiped into a point of the same dimensionality. 


\section{Background, related works, and motivation}

Generally, uncertainty of a group of $N$ parameters studied in $\mathbb{R}^{N}$ by $N \in \mathbb{N} \backslash\{1\}$ consists in that we can choose its value $\mathbf{X}$ from a subset $\mathbb{X} \subset \mathbb{R}^{N}$ :

$$
\mathbf{X}=\left[x_{k}\right]_{1 \times N} \in \underset{k=1}{X} \mathbb{X}_{k}=\mathbb{X} \subset \mathbb{R}^{N} \text { by }\left|\mathbb{X}_{n}\right|>1 \text { or } \mu_{\mathbb{R}}\left(\mathbb{X}_{n}\right) \neq 0 \forall n=\overline{1, N}
$$

If subset $\mathbb{X}$ is finite, then $\mu_{\mathbb{R}^{N}}(\mathbb{X})=0$ and only condition $\left|\mathbb{X}_{n}\right|>1 \forall n=\overline{1, N}$ in (1) remains relevant. Both conditions in (1) are relevant for infinite subset $\mathbb{X}$. The uncertainty becomes partial if $\mathbb{X}$ is a closed $N$-dimensional hyperparallelepiped within the nonnegative orthant of $\mathbb{R}^{N}$, and $\sum_{n=1}^{N} x_{n}$ is a constant value $[44,38]$. It is very convenient to consider the normalized uncertainty $[13,21,23,9]$ :

$$
\begin{gathered}
x_{k} \in\left[a_{k} ; b_{k}\right]=\mathbb{X}_{k} \subset(0 ; 1) \\
\text { by } a_{k}<b_{k} \forall k=\overline{1, N-1} \text { and } x_{N}=1-\sum_{n=1}^{N-1} x_{n}>0 .
\end{gathered}
$$

It is important to note that (2) is followed with an inequality

$$
\sum_{k=1}^{N-1} a_{k}<\sum_{k=1}^{N-1} b_{k}<1
$$

If we knew a probabilistic measure $\mathscr{F}_{n}\left(x_{n}\right)$ over $\mathbb{X}_{n}$ (being a generatrix to the Lebesgue-Stieltjes integral), where

$$
\int_{a_{n}}^{b_{n}} \mathrm{~d} \mathscr{F}_{n}\left(x_{n}\right)=1
$$

the $n$-th interval uncertainty could be reduced as follows:

$$
\mathrm{M}_{\mathscr{F}_{n}}(\Theta)=\int_{a_{n}}^{b_{n}} x_{n} \mathrm{~d} \mathscr{F}_{n}\left(x_{n}\right) .
$$

Value (4) is a point estimate [17], which is the mathematical expectation of a random variable $\Theta$ with its values $x_{n} \in\left[a_{n} ; b_{n}\right]$ by the probabilistic measure $\mathscr{F}_{n}\left(x_{n}\right)$. In particular, knowing a probability density function $f\left(x_{n}\right)$, which is $f_{n}\left(x_{n}\right) \mathrm{d} x_{n}=$ $\mathrm{d} \mathscr{F}_{n}\left(x_{n}\right)$, the point estimate becomes

$$
\mathrm{M}_{f_{n}}(\Theta)=\int_{a_{n}}^{b_{n}} x_{n} f_{n}\left(x_{n}\right) \mathrm{d} x_{n}
$$


However, as it was mentioned above, eliciting a probability function is not an easy process. All the more that there are simultaneously $N$ such functions (see, e.g., [29, $49]$ ), although the $N$-th function (for cases with $N>1$ ) is needless for the partial uncertainty, where value

$$
1-\sum_{n=1}^{N-1} \mathrm{M}_{\mathscr{F}_{n}}(\Theta) \quad \text { or } \quad 1-\sum_{n=1}^{N-1} \mathrm{M}_{f_{n}}(\Theta)
$$

can be accepted as a point estimate of the $N$-th interval. Besides, those $N-1$ probability functions must be constant through a given amount of time $[15,17,5$, 31]. Otherwise, the point estimates intended for this amount become fluent. Thus the estimation loses its sense.

Without statistical data, the guessed values are used instead of the intervals in (2). While "guessing", the minimax principle is applied in order to prevent the most inappropriate guess. The prevention is understood as smoothing over the most negative effect [40]. The effect is computed as a ratio between an aftermath of what is real (becoming known for us only after some period of time) and an aftermath of our guess (the point estimation aftermaths). Such aftermath is a function $\rho$ defined on every interval $[17,7,48,44]$. If we say that $y_{k}$ is a point estimate for the $k$-th interval but $x_{k}$ is a real value (valid for the given amount of time), then the effect is $\frac{\rho\left(x_{k}\right)}{\rho\left(y_{k}\right)}$. Owing to $(2)$, only $N-1$ point estimates

$$
\begin{aligned}
\mathbf{X}_{N-1} & =\left[x_{k}\right]_{1 \times(N-1)} \\
& \in\left\{\mathbf{X}_{N-1} \in \mathbb{R}^{N-1} \mid x_{n} \in\left[a_{n} ; b_{n}\right]=\mathbb{X}_{n} \subset(0 ; 1) \forall n=\overline{1, N-1}\right\}
\end{aligned}
$$

are guessed as

$$
\begin{aligned}
\mathbf{Y}_{N-1} & =\left[y_{k}\right]_{1 \times(N-1)} \\
& \in\left\{\mathbf{Y}_{N-1} \in \mathbb{R}^{N-1} \mid y_{m} \in\left[a_{m} ; b_{m}\right]=\mathbb{Y}_{m} \subset(0 ; 1) \forall m=\overline{1, N-1}\right\}
\end{aligned}
$$

Then the most negative effect is a function of $2 N-2$ variables:

$$
\begin{aligned}
K\left(\mathbf{X}_{N-1}, \mathbf{Y}_{N-1}\right) & =K\left(\left\{x_{i}\right\}_{i=1}^{N-1},\left\{y_{j}\right\}_{j=1}^{N-1}\right) \\
& =\max \left\{\left\{\frac{\rho\left(x_{k}\right)}{\rho\left(y_{k}\right)}\right\}_{k=1}^{N-1}, \frac{\rho\left(1-\sum_{n=1}^{N-1} x_{n}\right)}{\rho\left(1-\sum_{m=1}^{N-1} y_{m}\right)}\right\}
\end{aligned}
$$

In fact, (6) is a strategy of the decision-maker in the problem of choosing the best point estimates (Figure 1). The best version of this strategy is found as the second player's optimal strategy in a game with kernel (7) on a hyperparallelepiped 


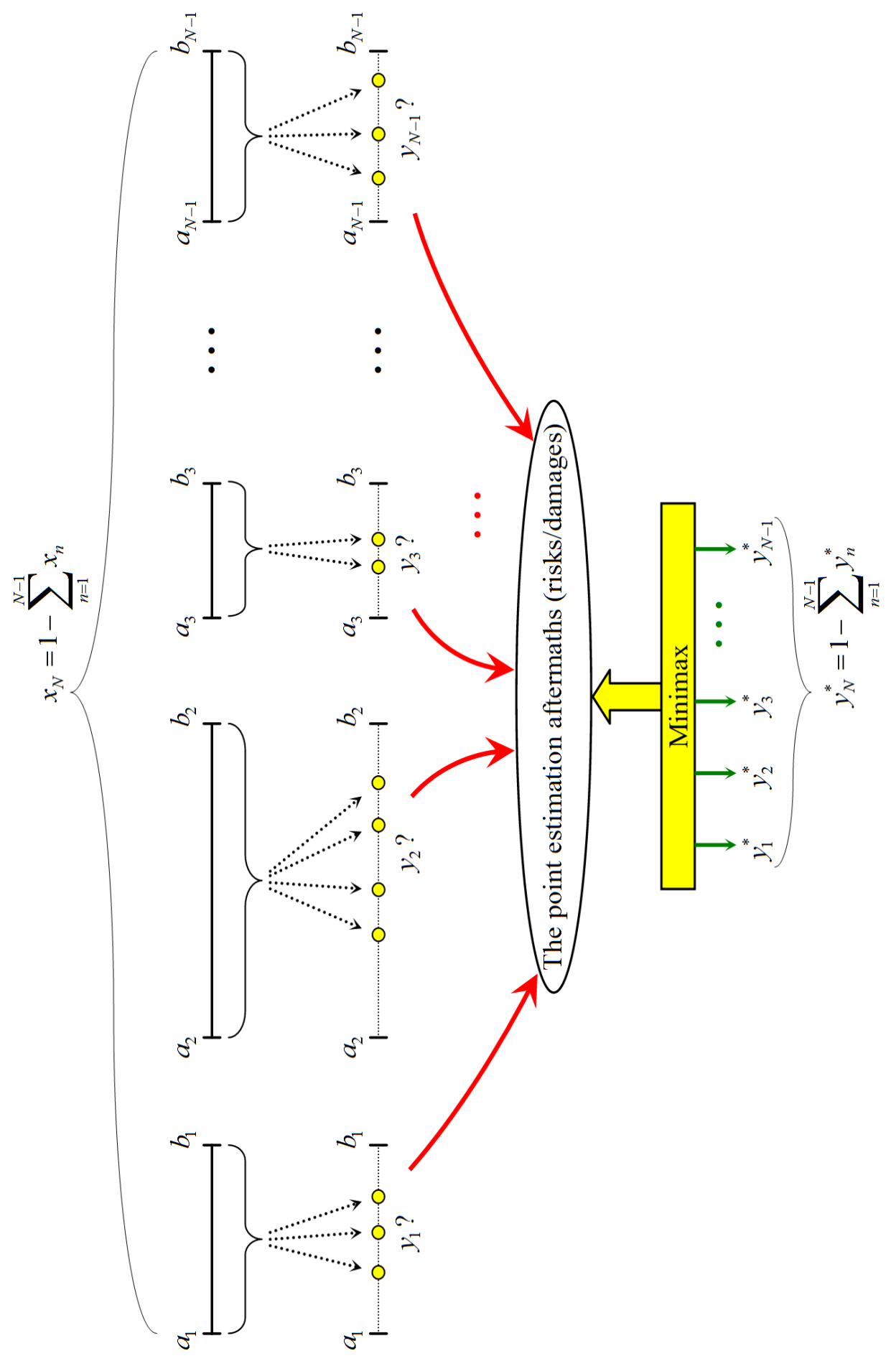

Figure 1: Conception of choosing the best point estimates with minimax 


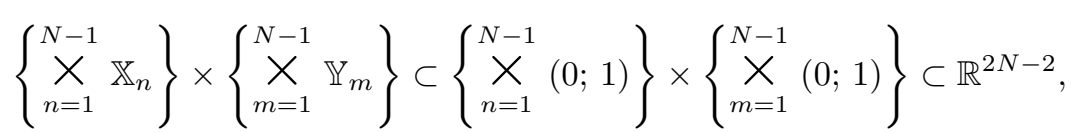

where (5) and (6) are pure strategies of the first and second players, respectively $[44,45]$. The first player's pure strategy set is $\underset{n=1}{\times} \mathbb{X}_{n}$, and the second player's pure strategy set is $\mathrm{X}_{m=1}^{N-1} \mathbb{Y}_{m}$.

The simplest case of the effect computation is just $\rho\left(y_{k}\right)=y_{k}($ see $[45,38])$. Then the game kernel (7) is

$$
\begin{aligned}
K\left(\mathbf{X}_{N-1}, \mathbf{Y}_{N-1}\right) & =K\left(\left\{x_{i}\right\}_{i=1}^{N-1},\left\{y_{j}\right\}_{j=1}^{N-1}\right) \\
& =\max \left\{\left\{\frac{x_{k}}{y_{k}}\right\}_{k=1}^{N-1}, \frac{1-\sum_{n=1}^{N-1} x_{n}}{1-\sum_{m=1}^{N-1} y_{m}}\right\} .
\end{aligned}
$$

In a trivial case, when $N=2$, kernel (9) is

$$
K\left(x_{1}, y_{1}\right)=\max \left\{\frac{x_{1}}{y_{1}}, \frac{1-x_{1}}{1-y_{1}}\right\}
$$

and the best minimax decision is [45]

$$
y_{1}^{*}=\frac{b_{1}}{1+b_{1}-a_{1}} .
$$

For the general case, in the game with kernel (9) on hyperparallelepiped (8), the decision-maker (as the second player) has a pure optimal strategy $\mathbf{Y}_{N-1}^{*}=$ $\left[y_{k}^{*}\right]_{1 \times(N-1)}($ see $[38])$.

The case with kernel (7) is still unsolved. Shall the decision-maker possess a pure optimal strategy in this case just for certain types of the function $\rho$ ? Will there be any peculiarities (or special cases) in computing components of strategy $\mathbf{Y}_{N-1}^{*}$ ? Can there be a continuum of such strategies? If it can, then what is a routine to select a single unique strategy? Answers to these open questions may become a fair contribution to the field of non-statistical interval uncertainty reduction.

\section{Goals and tasks to be fulfilled}

The goal is to find a decision-maker's optimal strategy $\mathbf{Y}_{N-1}^{*}$, if any, in the game with kernel

$$
K\left(\mathbf{X}_{N-1}, \mathbf{Y}_{N-1}\right)=K\left(\left\{x_{i}\right\}_{i=1}^{N-1},\left\{y_{j}\right\}_{j=1}^{N-1}\right)
$$




$$
=\max \left\{\left\{\frac{x_{k}^{q}}{y_{k}^{q}}\right\}_{k=1}^{N-1}, \frac{\left(1-\sum_{n=1}^{N-1} x_{n}\right)^{q}}{\left(1-\sum_{m=1}^{N-1} y_{m}\right)^{q}}\right\}
$$

on hyperparallelepiped (8) for any $q>0$. Along with point estimates $\left\{y_{m}^{*}\right\}_{m=1}^{N-1}$ of the first $N-1$ intervals, this strategy will allow to find a point estimate of the $N$-th interval as

$$
y_{N}^{*}=1-\sum_{m=1}^{N-1} y_{m}^{*} .
$$

For achieving the goal, the following tasks are to be fulfilled:

1. To ascertain whether the second player has an optimal pure strategy $\mathbf{Y}_{N-1}^{*}$ in the game.

2. If strategy $\mathbf{Y}_{N-1}^{*}$ exists, to state conditions for finding its components.

3. To give examples of finding components of strategy $\mathbf{Y}_{N-1}^{*}$.

4. To discuss its applicability and significance.

\section{Decision-maker's optimal strategy}

Before we get started, an abbreviation of the game notation should be given.

Definition 1. The game with kernel (10) on hyperparallelepiped (8) for any $q>0$ is called partial uncertainty reduction game (PURG).

The positive $q$ is not emphasized in PURG. The reason is going to be made plain below.

Theorem 1. The decision-maker has a pure optimal strategy

$$
\mathbf{Y}_{N-1}^{*}=\left[y_{k}^{*}\right]_{1 \times(N-1)}
$$

in PURG. Components of this strategy are

$$
y_{k}^{*}=\frac{b_{k}}{1+\sum_{n=1}^{N-1}\left(b_{n}-a_{n}\right)} \quad \forall k=\overline{1, N-1}
$$

if only

$$
\frac{b_{k}}{1+\sum_{n=1}^{N-1}\left(b_{n}-a_{n}\right)} \geqslant a_{k} \quad \forall k=\overline{1, N-1} .
$$


Proof. Let

$$
\begin{gathered}
L_{k}\left(x_{k}, y_{k}\right)=\frac{x_{k}^{q}}{y_{k}^{q}} \text { for } k=\overline{1, N-1} \text { and } \\
L_{N}\left(\left\{x_{i}\right\}_{i=1}^{N-1},\left\{y_{j}\right\}_{j=1}^{N-1}\right)=\frac{\left(1-\sum_{n=1}^{N-1} x_{n}\right)^{q}}{\left(1-\sum_{m=1}^{N-1} y_{m}\right)^{q}} .
\end{gathered}
$$

So, kernel (10) is a maximum of $N$ functions (14). Partial derivatives of the second order of those functions with respect to components of the second player's pure strategy are:

$$
\begin{aligned}
\frac{\partial^{2}}{\partial y_{k}^{2}} L_{k}\left(x_{k}, y_{k}\right) & =\frac{\partial^{2}}{\partial y_{k}^{2}}\left(\frac{x_{k}^{q}}{y_{k}^{q}}\right)=\frac{\partial}{\partial y_{k}}\left(-\frac{q x_{k}^{q}}{y_{k}^{q+1}}\right) \\
& =\frac{q(q+1) x_{k}^{q}}{y_{k}^{q+2}} \text { for } k=\overline{1, N-1}
\end{aligned}
$$

and

$$
\begin{aligned}
\frac{\partial^{2}}{\partial y_{k}^{2}} L_{N}\left(\left\{x_{i}\right\}_{i=1}^{N-1},\left\{y_{j}\right\}_{j=1}^{N-1}\right) & =\frac{\partial^{2}}{\partial y_{k}^{2}}\left(\frac{\left(1-\sum_{n=1}^{N-1} x_{n}\right)^{q}}{\left(1-\sum_{m=1}^{N-1} y_{m}\right)^{q}}\right) \\
& =\frac{\partial}{\partial y_{k}}\left(\frac{q\left(1-\sum_{n=1}^{N-1} x_{n}\right)^{q}}{\left(1-\sum_{m=1}^{N-1} y_{m}\right)^{q+1}}\right) \\
& =\frac{q(q+1)\left(1-\sum_{n=1}^{N-1} x_{n}\right)^{q}}{\left(1-\sum_{m=1}^{N-1} y_{m}\right)^{q+2}} \text { for } k=\overline{1, N-1 .} .
\end{aligned}
$$

Each of those $2 N-2$ derivatives (15) and (16) is positive. This implies that functions

$$
\left\{\left\{L_{k}\left(x_{k}, y_{k}\right)\right\}_{k=1}^{N-1}, L_{N}\left(\left\{x_{i}\right\}_{i=1}^{N-1},\left\{y_{j}\right\}_{j=1}^{N-1}\right)\right\}
$$

are strictly convex [44]. Then, owing to [37], function (10) is strictly convex itself. Therefore, PURG is convex. Owing to the game strict convexity, the second player 
herein has a pure strategy, which is

$$
\begin{aligned}
\mathbf{Y}_{N-1}^{*} \in & \arg \left\{\min _{\substack{N-1 \\
\mathbf{Y}_{N-1} \in \underset{m=1}{\times} \mathbb{Y}_{m}}}\left\{\max _{\mathbf{X}_{N-1} \in \underset{n=1}{\times} \mathbb{X}_{n}} K\left(\mathbf{X}_{N-1}, \mathbf{Y}_{N-1}\right)\right\}\right\} \\
= & \arg \left\{\min _{\mathbf{Y}_{N-1}^{N-1} \underset{m=1}{\times}\left[a_{m} ; b_{m}\right]}\left\{\max _{\substack{N-1 \\
\mathbf{X}_{N-1} \in \underset{n=1}{\times}\left[a_{n} ; b_{n}\right]}} K\left(\mathbf{X}_{N-1}, \mathbf{Y}_{N-1}\right)\right\} .\right.
\end{aligned}
$$

Further, we have:

$$
\begin{aligned}
& \max _{\mathbf{X}_{N-1}^{N-1} \in \underset{n}{\times}\left[a_{n} ; b_{n}\right]} K\left(\mathbf{X}_{N-1}, \mathbf{Y}_{N-1}\right)
\end{aligned}
$$

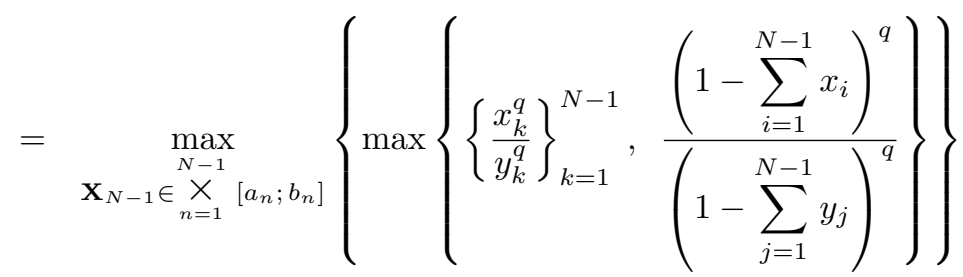

$$
\begin{aligned}
& =\max \left\{\left\{\max _{x_{k} \in\left[a_{k} ; b_{k}\right]}\left\{\frac{x_{k}^{q}}{y_{k}^{q}}\right\}\right\}_{k=1}^{N-1}, \quad \max _{\substack{N-1 \\
\mathbf{x}_{N-1} \in \underset{n=1}{\times}\left[a_{n} ; b_{n}\right]}}\left\{\frac{\left(1-\sum_{i=1}^{N-1} x_{i}\right)^{q}}{\left(1-\sum_{j=1}^{N-1} y_{j}\right)^{q}}\right\}\right. \\
& =\max \left\{\left\{\left(\frac{b_{k}}{y_{k}}\right)^{q}\right\}_{k=1}^{N-1},\left(\frac{1-\sum_{i=1}^{N-1} a_{i}}{1-\sum_{j=1}^{N-1} y_{j}}\right)^{q}\right\} \text {. }
\end{aligned}
$$

Maximum (17) is a function of $N-1$ variables $\left\{y_{k}\right\}_{k=1}^{N-1}$. This function consists of $N$ upper parts of hyperbolic hypersurfaces raised to the power $q$. Its minimum on $N-1$ $\underset{m=1}{\times} \mathbb{Y}_{m}$ is reached when all those $N$ parts are equal $[45,38]$ :

$$
\min _{\mathbf{Y}_{N-1} \in{\underset{m=1}{N-1}}_{m}^{\times}\left[a_{m} ; b_{m}\right]}\left\{\max _{\mathbf{X}_{N-1} \in \underset{n=1}{\times}\left[a_{n} ; b_{n}\right]} K\left(\mathbf{X}_{N-1}, \mathbf{Y}_{N-1}\right)\right\}
$$




$$
\begin{aligned}
& =\min _{\substack{N-1 \\
\mathbf{Y}_{N-1} \in \underset{m=1}{\times}\left[a_{m} ; b_{m}\right]}}\left\{\max \left\{\left\{\left(\frac{b_{k}}{y_{k}}\right)^{q}\right\}_{k=1}^{N-1},\left(\frac{1-\sum_{i=1}^{N-1} a_{i}}{1-\sum_{j=1}^{N-1} y_{j}}\right)^{q}\right\}\right\} \\
& =\max \left\{\left\{\left(\frac{b_{k}}{y_{k}^{*}}\right)^{q}\right\}_{k=1}^{N-1},\left(\frac{1-\sum_{i=1}^{N-1} a_{i}}{1-\sum_{j=1}^{N-1} y_{j}^{*}}\right)^{q}\right\}=v_{\mathrm{opt}},
\end{aligned}
$$

where the optimal game value

$$
v_{\mathrm{opt}}=\left(\frac{b_{k}}{y_{k}^{*}}\right)^{q}=\left(\frac{1-\sum_{i=1}^{N-1} a_{i}}{1-\sum_{j=1}^{N-1} y_{j}^{*}}\right)^{q} \quad \forall k=\overline{1, N-1} .
$$

It is apparent that we can state

$$
\frac{b_{k}}{y_{k}^{*}}=\frac{1-\sum_{i=1}^{N-1} a_{i}}{1-\sum_{j=1}^{N-1} y_{j}^{*}} \quad \forall k=\overline{1, N-1}
$$

instead of (18). It follows from (18) that

$$
y_{m}^{*}=\frac{b_{m}}{b_{k}} y_{k}^{*} \quad \forall m=\overline{1, N-1} \quad \text { and } \quad \forall k=\overline{1, N-1}
$$

along with that

$$
y_{k}^{*}\left(1-\sum_{i=1}^{N-1} a_{i}\right)=b_{k}\left(1-\sum_{j=1}^{N-1} y_{j}^{*}\right) \quad \forall k=\overline{1, N-1} .
$$

Ratio (20) allows to see that

$$
b_{k} y_{m}^{*}=b_{k} \frac{b_{m}}{b_{k}} y_{k}^{*}=b_{m} y_{k}^{*} .
$$

Equality (22) implies that we can exchange indices. This allows to re-write the right term in (21) as follows:

$$
b_{k}\left(1-\sum_{j=1}^{N-1} y_{j}^{*}\right)=b_{k}-\sum_{j=1}^{N-1} b_{k} y_{j}^{*}=b_{k}-\sum_{j=1}^{N-1} b_{j} y_{k}^{*}=b_{k}-y_{k}^{*} \sum_{j=1}^{N-1} b_{j} .
$$


Now, we plug the last term of (23) into the right-hand side of (21):

$$
y_{k}^{*}\left(1-\sum_{i=1}^{N-1} a_{i}\right)=b_{k}-y_{k}^{*} \sum_{j=1}^{N-1} b_{j},
$$

whence

$$
y_{k}^{*}=\frac{b_{k}}{1+\sum_{j=1}^{N-1} b_{j}-\sum_{i=1}^{N-1} a_{i}}=\frac{b_{k}}{1+\sum_{n=1}^{N-1}\left(b_{n}-a_{n}\right)} .
$$

However, value (12) can really be the $k$-th component of the decision-maker's optimal strategy if

$$
\frac{b_{k}}{1+\sum_{n=1}^{N-1}\left(b_{n}-a_{n}\right)} \in\left[a_{k} ; b_{k}\right]
$$

Inequality

$$
\frac{b_{k}}{1+\sum_{n=1}^{N-1}\left(b_{n}-a_{n}\right)} \leqslant b_{k} \quad \forall k=\overline{1, N-1}
$$

is always true because

$$
\frac{1}{1+\sum_{n=1}^{N-1}\left(b_{n}-a_{n}\right)} \leqslant 1
$$

and

$$
1 \leqslant 1+\sum_{n=1}^{N-1}\left(b_{n}-a_{n}\right)
$$

where

$$
\sum_{n=1}^{N-1}\left(b_{n}-a_{n}\right)>0
$$

that follows (3). Finally, just requirement (13) remains.

Thus the decision-maker's optimal strategy does not depend upon $q$. The optimal game value (18), showing the poorest ratio between the point estimation aftermaths, however, is directly influenced with $q$.

Example 1. Consider an example construing the sense of requirement (13). Suppose that $N=3, a_{1}=0.3, b_{1}=0.6, a_{2}=0.25, b_{2}=0.3$. Then

$$
\frac{b_{1}}{1+\sum_{n=1}^{2}\left(b_{n}-a_{n}\right)}=\frac{0.6}{1+0.6-0.3+0.3-0.25}=\frac{4}{9}>\frac{3}{10}=a_{1}
$$


but

$$
\frac{b_{2}}{1+\sum_{n=1}^{2}\left(b_{n}-a_{n}\right)}=\frac{0.3}{1+0.6-0.3+0.3-0.25}=\frac{2}{9}<\frac{1}{4}=a_{2} .
$$

Thus requirement (13) is violated here for the second component. This counterexample prompts to consider the following theorem of necessity.

Theorem 2. To have the $k$-th component as (12) in PURG, it is necessary that the inequality

$$
\frac{b_{k}-a_{k}}{\sum_{n=1}^{N-1}\left(b_{n}-a_{n}\right)} \geqslant a_{k}
$$

be true.

Proof. Requirement (13) for the $k$-th component is expanded as follows:

$$
\begin{gathered}
b_{k} \geqslant a_{k}+a_{k} \sum_{n=1}^{N-1}\left(b_{n}-a_{n}\right), \\
b_{k}-a_{k} \geqslant a_{k}\left(\sum_{m=1}^{N-1} b_{m}-\sum_{m=1}^{N-1} a_{m}\right),
\end{gathered}
$$

whence, dividing with owing to (3), we get inequality (27).

It will be shown below that condition (27) is not sufficient for having the $k$-th component as (12). But it is sufficient to say that if condition (27) is violated, then the $k$-th component is not computed as (12). This is, so to speak, a rejection of conditions in Theorem 1. Henceforward, the following definitions become important.

Definition 2. The second player's optimal strategy $\mathbf{Y}_{N-1}^{*}$ in PURG is called regular if its components are computed as (12). A component of a regular strategy is called regular.

Definition 3. The regular optimal strategy $\mathbf{Y}_{N-1}^{*}$ in PURG is called totally regular if

$$
y_{k}^{*} \in\left(a_{k} ; b_{k}\right) \quad \forall k=\overline{1, N-1} .
$$

A component of a totally regular strategy is called totally regular.

Definition 4. The second player's optimal strategy $\mathbf{Y}_{N-1}^{*}$ in PURG is called irregular if at least a one inequality in requirement (13) is violated. A component of an irregular strategy is called irregular.

These definitions facilitate in treating various types of optimal strategy $\mathbf{Y}_{N-1}^{*}$. It easy to see that if inequality (27) holds for every $k=\overline{1, N-1}$, then $\mathbf{Y}_{N-1}^{*}$ is regular. Obviously, a regular strategy $\mathbf{Y}_{N-1}^{*}$ consists of $N-1$ (all) regular components. They are computed straightforwardly by (12). If strategy $\mathbf{Y}_{N-1}^{*}$ has an irregular component, this strategy is not a regular one. Before finding irregular strategies, the span of the regular component is asserted below. 
Theorem 3. Whichever regular strategy $\mathbf{Y}_{N-1}^{*}$ in PURG is, its $k$-th component

$$
y_{k}^{*} \in\left[a_{k} ; b_{k}\right) \quad \forall k=\overline{1, N-1} .
$$

Proof. As an inequality in requirement (13) can be violated, then an occurrence $y_{k}^{*}=a_{k}$ is possible. Owing to (26), inequality (25) holds strictly. Hence, inequality (24) holds strictly as well.

Do we know a PURG, in which strategy $\mathbf{Y}_{N-1}^{*}$ is totally regular? Actually, such a PURG exists, although the intervals seem "regular" themselves [38].

Theorem 4. In PURG whose hyperparallelepiped (8) is a hypercube

$$
\begin{aligned}
& \left\{{\underset{n=1}{X}}_{\mathrm{X}}^{N-1}[a ; b]\right\} \times\{\underset{m=1}{\mathrm{X}}[a ; b]\} \subset\{\underset{n=1}{\mathrm{X}}(0 ; 1)\} \times\{\underset{m=1}{\mathrm{X}}(0 ; 1)\} \subset \mathbb{R}^{2 N-2} \\
& \text { by } \\
& \left\{\left[a_{k} ; b_{k}\right]=[a ; b]\right\}_{k=1}^{N-1},
\end{aligned}
$$

the decision-maker has a totally regular strategy $\mathbf{Y}_{N-1}^{*}$ whose components are identical:

$$
y_{k}^{*}=\frac{b}{1+(N-1)(b-a)} \quad \forall k=\overline{1, N-1} .
$$

Proof. Formula (32) is directly obtained by plugging (31) into (12). We know from (3) that

$$
\sum_{k=1}^{N-1} a_{k}=(N-1) a<1 .
$$

So,

$$
\frac{1}{N-1}>a
$$

and

$$
\frac{b-a}{(N-1)(b-a)}>a \text {. }
$$

Inequality (33) is referred to Theorem 2 by considering inequality (27) with the strict sign that gives (28).

Thus identical or "regular" intervals (31) generate totally regular and identical components (32). In this way, interval uncertainties are "regulated" if we are allowed to slightly adjust endpoints of different intervals. Of course, this is not always possible in practical situations. If endpoints of different intervals are not adjustable, then condition (13) is not guaranteed. Now the question is whether requirement (13) can be violated entirely, i.e., inequality (27) fails $\forall k=\overline{1, N-1}$. 
Theorem 5. Inequality (27) in PURG holds strictly at least for a one $k \in\{\overline{1, N-1}\}$.

Proof. Assume that inequality (27) nonstrictly fails $\forall k=\overline{1, N-1}$. This implies that

$$
\frac{b_{k}-a_{k}}{\sum_{n=1}^{N-1}\left(b_{n}-a_{n}\right)} \leqslant a_{k} \quad \forall k=\overline{1, N-1 .}
$$

Then, summing up both sides, we get

$$
\frac{\sum_{k=1}^{N-1}\left(b_{k}-a_{k}\right)}{\sum_{n=1}^{N-1}\left(b_{n}-a_{n}\right)}=1 \leqslant \sum_{k=1}^{N-1} a_{k}
$$

that is impossible. This contradiction proves the theorem assertion.

So, strategy $\mathbf{Y}_{N-1}^{*}$ is irregular if inequality (27) fails at least for a one $k \in$ $\{\overline{1, N-1}\}$. How is $y_{k}^{*}$ then computed? What is strategy $\mathbf{Y}_{N-1}^{*}$ then, after all? The following theorem answers this question partially.

Theorem 6. In PURG, let

$$
\frac{b_{u}}{1+\sum_{n=1}^{N-1}\left(b_{n}-a_{n}\right)}<a_{u} \quad \text { for } \quad u \in \mathscr{U} \subset\{\overline{1, N-1}\} \quad \text { by } \quad \mathscr{U} \neq \emptyset .
$$

Then $|\mathscr{U}|$ components of an irregular strategy $\mathbf{Y}_{N-1}^{*}$ are found as

$$
y_{u}^{*}=a_{u} \quad \forall u \in \mathscr{U} \subset\{\overline{1, N-1}\} .
$$

The rest $N-1-|\mathscr{U}|$ components are computed as

$$
y_{k}^{*}=\frac{b_{k}\left(1-\sum_{w \in \mathscr{U}} a_{w}\right)}{1+\sum_{n=1}^{N-1}\left(b_{n}-a_{n}\right)-\sum_{w \in \mathscr{U}} b_{w}} \quad \forall k \in\{\overline{1, N-1}\} \backslash \mathscr{U}
$$

if only

$$
\frac{b_{k}\left(1-\sum_{w \in \mathscr{U}} a_{w}\right)}{1+\sum_{n=1}^{N-1}\left(b_{n}-a_{n}\right)-\sum_{w \in \mathscr{U}} b_{w}} \geqslant a_{k} \quad \forall k \in\{\overline{1, N-1}\} \backslash \mathscr{U} .
$$


Proof. Let

$$
y_{u}^{* *}=\frac{b_{u}}{1+\sum_{n=1}^{N-1}\left(b_{n}-a_{n}\right)} \quad \text { for } \quad u \in \mathscr{U} \subset\{\overline{1, N-1}\}
$$

Due to (34), points (38) cannot be components of $\mathbf{Y}_{N-1}^{*}$ because $y_{u}^{* *} \notin\left[a_{u} ; b_{u}\right]$. So,

$$
y_{u}^{*}>y_{u}^{* *} \quad \forall u \in \mathscr{U} .
$$

Let (18) be re-written as

$$
\begin{gathered}
\left(\frac{b_{u}}{y_{u}^{* *}}\right)^{q}=\left(\frac{b_{k}}{y_{k}^{*}}\right)^{q}=\left(\frac{1-\sum_{i=1}^{N-1} a_{i}}{1-\sum_{z \in\left\{\frac{1, N-1}{1-1} y_{z}^{*}-\sum_{w \in \mathscr{U}} y_{w}^{* *}\right.}}\right)^{q} \\
\forall u \in \mathscr{U} \text { and } \forall k \in\{\overline{1, N-1}\} \backslash \mathscr{U} .
\end{gathered}
$$

Taking into account (39), from (40) we get

$$
\begin{gathered}
\left(\frac{b_{u}}{y_{u}^{*}}\right)^{q}<\left(\frac{b_{k}}{y_{k}^{*}}\right)^{q}<\left(\frac{1-\sum_{i=1}^{N-1} a_{i}}{1-\sum_{z \in\left\{\frac{1, N-1}{1, \backslash \mathscr{U}} y_{z}^{*}-\sum_{w \in \mathscr{U}} y_{w}^{*}\right.}}\right)^{q} \\
\forall u \in \mathscr{U} \text { and } \forall k \in\{\overline{1, N-1}\} \backslash \mathscr{U}
\end{gathered}
$$

instead of (18). Inequalities (41) are equivalent to inequalities

$$
\begin{gathered}
\frac{b_{u}}{y_{u}^{*}}<\frac{b_{k}}{y_{k}^{*}}<\frac{1-\sum_{i=1}^{N-1} a_{i}}{1-\sum_{z \in\{\overline{1, N-1}\} \backslash \mathscr{U}} y_{z}^{*}-\sum_{w \in \mathscr{U}} y_{w}^{*}} \\
\forall u \in \mathscr{U} \text { and } \forall k \in\{\overline{1, N-1}\} \backslash \mathscr{U} .
\end{gathered}
$$

Similarly to getting equality (19), the second player here endeavors to minimize the right term in (42) to get equality

$$
v_{\mathrm{opt}}=\frac{b_{k}}{y_{k}^{*}}=\frac{1-\sum_{i=1}^{N-1} a_{i}}{1-\sum_{z \in\{1, N-1}^{1, \backslash \mathscr{U}} y_{z}^{*}-\sum_{w \in \mathscr{U}} y_{w}^{*}} \quad \forall k \in\{\overline{1, N-1}\} \backslash \mathscr{U}
$$


that is still possible by setting

$$
y_{k}^{*}<\frac{b_{k}}{1+\sum_{n=1}^{N-1}\left(b_{n}-a_{n}\right)} \quad \text { for } \frac{b_{k}}{1+\sum_{n=1}^{N-1}\left(b_{n}-a_{n}\right)}>a_{k} .
$$

Setting simultaneously $y_{w}^{*}>a_{w}$ only increases the right term in (43). To decrease this term, it would be necessary to decrease $y_{k}^{*}$ for some $k \in\{\overline{1, N-1}\} \backslash \mathscr{U}$ by (44) more. But then the term $\frac{b_{k}}{y_{k}^{*}}$ would be increased as well. Such behavior of the second player contradicts with its optimality principle, where $v_{\text {opt }}$ is tried for minimization. Therefore, setting (35) is optimal, and the rest $N-1-|\mathscr{U}|$ components are roots of equations (43). Similarly to (20),

$$
y_{m}^{*}=\frac{b_{m}}{b_{k}} y_{k}^{*} \forall m \in\{\overline{1, N-1}\} \backslash \mathscr{U} \quad \text { and } \quad \forall k \in\{\overline{1, N-1}\} \backslash \mathscr{U},
$$

whence exchanging indices $(22)$ is true. On the other hand, we get $N-1-|\mathscr{U}|$ equations

$$
\begin{aligned}
& y_{k}^{*}\left(1-\sum_{i=1}^{N-1} a_{i}\right) \\
& =b_{k}\left(1-\sum_{z \in\left\{\frac{1, N-1}{1, N} \backslash \mathscr{U}\right.} y_{z}^{*}-\sum_{w \in \mathscr{U}} y_{w}^{*}\right) \quad \forall k \in\{\overline{1, N-1}\} \backslash \mathscr{U}
\end{aligned}
$$

from (43). Equations (46) are re-written with (35) and (45) as follows:

$$
\begin{aligned}
b_{k}\left(1-\sum_{z \in\{1, N-1\} \backslash \mathscr{U}} y_{z}^{*}-\sum_{w \in \mathscr{U}} y_{w}^{*}\right) \\
=b_{k}\left(1-\sum_{w \in \mathscr{U}} a_{w}\right)-b_{k} \sum_{z \in\left\{\frac{1, N-1}{1,}\right\} \backslash \mathscr{U}} y_{z}^{*} \\
=b_{k}\left(1-\sum_{w \in \mathscr{U}} a_{w}\right)-y_{k}^{*} \sum_{z \in\{1, N-1} b_{z} \\
=b_{k}\left(1-\sum_{w \in \mathscr{U}} a_{w}\right)-y_{k}^{*}\left(\sum_{i=1}^{N-1} b_{i}-\sum_{w \in \mathscr{U}} b_{w}\right) \quad \forall k \in\{\overline{1, N-1}\} \backslash \mathscr{U} .
\end{aligned}
$$

Now, we plug the last term of (47) into the right-hand side of (46):

$$
y_{k}^{*}\left(1-\sum_{i=1}^{N-1} a_{i}\right)
$$




$$
=b_{k}\left(1-\sum_{w \in \mathscr{U}} a_{w}\right)-y_{k}^{*}\left(\sum_{i=1}^{N-1} b_{i}-\sum_{w \in \mathscr{U}} b_{w}\right) \quad \forall k \in\{\overline{1, N-1}\} \backslash \mathscr{U},
$$

whence (36) follows on. However, value (36) can really be the $k$-th component of the decision-maker's optimal strategy if

$$
\frac{b_{k}\left(1-\sum_{w \in \mathscr{U}} a_{w}\right)}{1+\sum_{n=1}^{N-1}\left(b_{n}-a_{n}\right)-\sum_{w \in \mathscr{U}} b_{w}} \in\left[a_{k} ; b_{k}\right] \quad \forall k \in\{\overline{1, N-1}\} \backslash \mathscr{U}
$$

From inequality

$$
\frac{b_{k}\left(1-\sum_{w \in \mathscr{U}} a_{w}\right)}{1+\sum_{n=1}^{N-1}\left(b_{n}-a_{n}\right)-\sum_{w \in \mathscr{U}} b_{w}} \leqslant b_{k} \quad \forall k \in\{\overline{1, N-1}\} \backslash \mathscr{U}
$$

successively we have:

$$
\begin{gathered}
\frac{1-\sum_{w \in \mathscr{U}} a_{w}}{1+\sum_{n=1}^{N-1}\left(b_{n}-a_{n}\right)-\sum_{w \in \mathscr{U}} b_{w}} \leqslant 1, \\
1-\sum_{w \in \mathscr{U}} a_{w} \leqslant 1+\sum_{n=1}^{N-1}\left(b_{n}-a_{n}\right)-\sum_{w \in \mathscr{U}} b_{w}, \\
0 \leqslant \sum_{n=1}^{N-1}\left(b_{n}-a_{n}\right)-\sum_{w \in \mathscr{U}}\left(b_{w}-a_{w}\right)=\sum_{z \in\left\{\frac{1, N-1}{1, N} \backslash \mathscr{U}\right.}\left(b_{z}-a_{z}\right) .
\end{gathered}
$$

Inequality (49) is always true, so inequality (48) holds as well. Finally, just requirement (37) remains.

According to Theorem 5, in PURG $\exists k \in\{\overline{1, N-1}\}$ such that the $k$-th inequality in requirement (13) holds. Therefore, the maximal number of inequalities (34) is $N-2$, i.e., $|\mathscr{U}| \leqslant N-2$. So, Example 1 gave a case with the maximal number by $N=3$, where $\mathscr{U}=\{2\}$. For that case, $y_{2}^{*}=a_{2}=0.25$ and

$$
y_{1}^{*}=\frac{b_{1}\left(1-a_{2}\right)}{1+\sum_{n=1}^{2}\left(b_{n}-a_{n}\right)-b_{2}}=\frac{0.6 \cdot 0.75}{1+0.6-0.3+0.3-0.25-0.3}=\frac{3}{7}>\frac{3}{10}=a_{1} .
$$


Example 2. Consider an example construing the sense of requirement (37). Suppose that $N=4, a_{1}=0.1, b_{1}=0.5, a_{2}=0.15, b_{2}=0.2, a_{3}=0.16, b_{3}=0.25$. Then

$$
\begin{gathered}
\sum_{n=1}^{3}\left(b_{n}-a_{n}\right)=0.5-0.1+0.2-0.15+0.25-0.16=0.54 \\
\frac{b_{1}}{1+\sum_{n=1}^{3}\left(b_{n}-a_{n}\right)}=\frac{0.5}{1+0.54}=\frac{25}{77}>\frac{1}{10}=a_{1}
\end{gathered}
$$

and

$$
\frac{b_{3}}{1+\sum_{n=1}^{3}\left(b_{n}-a_{n}\right)}=\frac{0.25}{1+0.54}=\frac{25}{154}>\frac{4}{25}=a_{3},
$$

but

$$
\frac{b_{2}}{1+\sum_{n=1}^{3}\left(b_{n}-a_{n}\right)}=\frac{0.2}{1+0.54}=\frac{10}{77}<\frac{3}{20}=a_{2} .
$$

So, $\mathscr{U}=\{2\}$ and, according to Theorem $6, y_{2}^{*}=a_{2}=0.15$,

$$
\frac{b_{1}\left(1-a_{2}\right)}{1+\sum_{n=1}^{3}\left(b_{n}-a_{n}\right)-b_{2}}=\frac{0.5 \cdot(1-0.15)}{1+0.54-0.2}=\frac{85}{268}>\frac{1}{10}=a_{1},
$$

but

$$
\frac{b_{3}\left(1-a_{2}\right)}{1+\sum_{n=1}^{3}\left(b_{n}-a_{n}\right)-b_{2}}=\frac{0.25 \cdot(1-0.15)}{1+0.54-0.2}=\frac{85}{536}<\frac{4}{25}=a_{3} .
$$

Thus requirement (37) is violated here for the third component. This counterexample shows that Theorem 6 does not conclude the question of finding an irregular strategy $\mathbf{Y}_{N-1}^{*}$ in PURG. This question also needs knowing the span of the irregular strategy component.

Theorem 7. Whichever irregular strategy $\mathbf{Y}_{N-1}^{*}$ in PURG is, its $k$-th component is (29). Besides,

$$
\begin{gathered}
\frac{b_{k}\left(1-\sum_{w \in \mathscr{U}} a_{w}\right)}{1+\sum_{n=1}^{N-1}\left(b_{n}-a_{n}\right)-\sum_{w \in \mathscr{U}} b_{w} \quad 1+\sum_{n=1}^{N-1}\left(b_{n}-a_{n}\right)}<b_{k} \\
\forall k \in\{\overline{1, N-1}\} \backslash \mathscr{U} .
\end{gathered}
$$


Proof. Let components of an irregular strategy $\mathbf{Y}_{N-1}^{*}$ be (35) and (36). The right inequality in (50) has been already proved in Theorem 3. Consider the difference between the left and right terms in the left inequality in (50) divided by $b_{k}$ :

$$
\begin{aligned}
\left(1-\sum_{w \in \mathscr{U}} a_{w}\right)\left(1+\sum_{n=1}^{N-1}\left(b_{n}-a_{n}\right)\right)-\left(1+\sum_{n=1}^{N-1}\left(b_{n}-a_{n}\right)-\sum_{w \in \mathscr{U}} b_{w}\right) & \left(1+\sum_{n=1}^{N-1}\left(b_{n}-a_{n}\right)-\sum_{w \in \mathscr{U}} b_{w}\right)\left(1+\sum_{n=1}^{N-1}\left(b_{n}-a_{n}\right)\right) \\
= & \left(1+\sum_{n=1}^{N-1}\left(b_{n}-a_{n}\right)-\sum_{w \in U} a_{w}\left(1+\sum_{n=1}^{N-1}\left(b_{n}-a_{n}\right)\right)-1-\sum_{n=1}^{N-1}\left(b_{n}-a_{n}\right)\right. \\
+ & \left.\sum_{w \in U} b_{w}\right)\left(1+\sum_{n=1}^{N-1}\left(b_{n}-a_{n}\right)-\sum_{w \in U} b_{w}\right)^{-1}\left(1+\sum_{n=1}^{N-1}\left(b_{n}-a_{n}\right)\right)^{-1} \\
= & \frac{-\sum_{w \in \mathscr{U}} a_{w}\left(1+\sum_{n=1}^{N-1}\left(b_{n}-a_{n}\right)\right)^{N-1} \sum_{w \in \mathscr{U}}^{N-1} b_{w}}{\left(1+\sum_{n=1}^{N-1}\left(b_{n}-a_{n}\right)-\sum_{w \in \mathscr{U}} b_{w}\right)\left(1+\sum_{n=1}^{N}\left(b_{n}-a_{n}\right)\right)} .
\end{aligned}
$$

Summing up both sides of (34), we get

$$
\begin{gathered}
\frac{\sum_{u \in \mathscr{U}} b_{u}}{1+\sum_{n=1}^{N-1}\left(b_{n}-a_{n}\right)}<\sum_{u \in \mathscr{U}} a_{u}, \\
\sum_{u \in \mathscr{U}} b_{u}-\sum_{u \in \mathscr{U}} a_{u}\left(1+\sum_{n=1}^{N-1}\left(b_{n}-a_{n}\right)\right)<0,
\end{gathered}
$$

whence the numerator in the last term of (51) is negative. This confirms the double inequality (50).

As requirement (37) can be violated at least for a one $k$, irregularity of strategy $\mathbf{Y}_{N-1}^{*}$ by Theorem 6 and property (50) of span (29) of its components need supplementation. An irregular strategy $\mathbf{Y}_{N-1}^{*}$, wherein

$$
\frac{b_{k}\left(1-\sum_{w \in \mathscr{U}} a_{w}\right)}{1+\sum_{n=1}^{N-1}\left(b_{n}-a_{n}\right)-\sum_{w \in \mathscr{U}} b_{w}}<a_{k} \quad \text { by } \quad k \in\{\overline{1, N-1}\} \backslash \mathscr{U},
$$


acquires deeper irregularity. Therefore, components of the irregular strategy should be distinguished by ranks of their irregularity. Particularly, this is about downwardbiasing of their values in accordance to (50).

Definition 5. The $u$-th component (35) of the second player's optimal strategy $\mathbf{Y}_{N-1}^{*}$ in PURG is called a simple irregular component of the first rank (SIC-1) if condition (34) holds. A set of those components (SICs-1) is called a left strategy subset of the first rank (LSS-1).

Definition 6. The $k$-th component (36) of the second player's optimal strategy $\mathbf{Y}_{N-1}^{*}$ in PURG is called a downward-biased irregular component of the first rank (DBIC-1). A set of those components (DBICs-1) is called a biased strategy subset of the first rank (BSS-1).

Clearly, an irregular strategy $\mathbf{Y}_{N-1}^{*}$ contains at least an SIC-1. Owing to Theorem 5 we know that number of SICs-1 does not exceed $N-2$. Going deeper with irregularity, can requirement (37) be violated entirely?

Theorem 8. Whichever nonempty LSS-1 in PURG is, $\exists l \in\{\overline{1, N-1}\} \backslash \mathscr{U}$ such that

$$
\frac{b_{l}\left(1-\sum_{w \in \mathscr{U}} a_{w}\right)}{1+\sum_{n=1}^{N-1}\left(b_{n}-a_{n}\right)-\sum_{w \in \mathscr{U}} b_{w}}>a_{l} .
$$

Proof. Assume that inequality (37) holds with the reverse sign implying that

$$
\frac{b_{k}\left(1-\sum_{w \in \mathscr{U}} a_{w}\right)}{1+\sum_{n=1}^{N-1}\left(b_{n}-a_{n}\right)-\sum_{w \in \mathscr{U}} b_{w}} \leqslant a_{k} \quad \forall k \in\{\overline{1, N-1}\} \backslash \mathscr{U} .
$$

Then, summing up both sides of (53) over $k \in\{\overline{1, N-1}\} \backslash \mathscr{U}$, we get

$$
\begin{aligned}
& \frac{\sum_{k \in\{1, N-1\} \backslash \mathscr{U}} b_{k}\left(1-\sum_{w \in \mathscr{U}} a_{w}\right)}{1+\sum_{n=1}^{N-1}\left(b_{n}-a_{n}\right)-\sum_{w \in \mathscr{U}} b_{w}} \leqslant \sum_{k \in\{1, N-1\} \backslash \mathscr{U}} a_{k},
\end{aligned}
$$

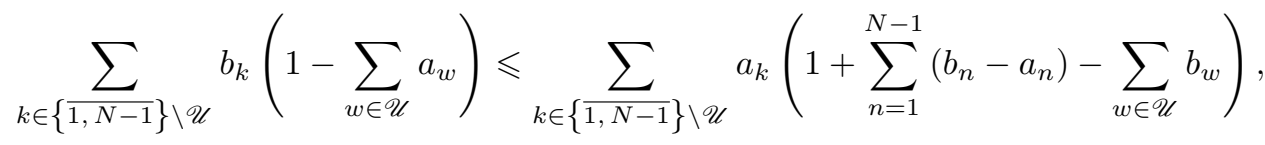




$$
\begin{aligned}
& \sum_{k \in\left\{\frac{1, N-1}{1, \backslash \mathscr{U}}\right.} b_{k}\left(1-\sum_{w \in \mathscr{U}} a_{w}\right) \\
& \leqslant \sum_{k \in\{1, N-1\} \backslash \mathscr{U}} a_{k}\left(1-\sum_{n=1}^{N-1} a_{n}+\sum_{j \in\{1, N-1\} \backslash \mathscr{U}} b_{j}\right), \\
& \sum_{k \in\left\{\frac{1, N-1}{1, N} \backslash \mathscr{U}\right.} b_{k}\left(1-\sum_{w \in \mathscr{U}} a_{w}\right)-\sum_{k \in\left\{\frac{1, N-1}{1, N} \backslash \mathscr{U}\right.} a_{k} \sum_{j \in\left\{\frac{1, N-1}{1, N} \backslash \mathscr{U}\right.} b_{j} \\
& \leqslant \sum_{k \in\left\{\frac{1, N-1}{1,} \backslash \mathscr{U}\right.} a_{k}\left(1-\sum_{n=1}^{N-1} a_{n}\right), \\
& \sum_{k \in\{1, N-1\} \backslash \mathscr{U}} b_{k}\left(1-\sum_{w \in \mathscr{U}} a_{w}-\sum_{k \in\{1, N-1\} \backslash \mathscr{U}} a_{k}\right)
\end{aligned}
$$

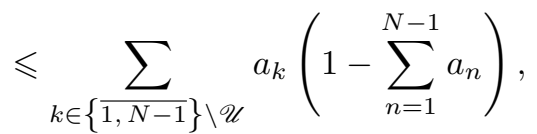

$$
\begin{aligned}
& \sum_{k \in\left\{\frac{1, N-1}{1,1} \backslash \mathscr{U}\right.} b_{k}\left(1-\sum_{n=1}^{N-1} a_{n}\right) \leqslant \sum_{k \in\left\{\frac{1, N-1}{1, N} \backslash \mathscr{U}\right.} a_{k}\left(1-\sum_{n=1}^{N-1} a_{n}\right), \\
& \sum_{k \in\{1, N-1\} \backslash \mathscr{U}} b_{k} \leqslant \sum_{k \in\{1, N-1\} \backslash \mathscr{U}} a_{k},
\end{aligned}
$$

that is impossible. The refuted assumption implies that $\exists l \in\{\overline{1, N-1}\} \backslash \mathscr{U}$ such that (52) holds.

Although requirement (37) cannot be violated entirely, some of its inequalities may turn false (see Example 2). Then the corresponding irregular optimal strategy $\mathbf{Y}_{N-1}^{*}$ does not contain BSS-1.

Definition 7. An irregular optimal strategy $\mathbf{Y}_{N-1}^{*}$ of the second player in PURG is called an irregular strategy of the first rank (IS-1) if it consists only of LSS-1 and BSS-1.

We found an IS-1 in Example 1. Example 2 showed a case, where DBICs-1 cannot be computed by (36), although $y_{2}^{*}=0.15$ turned to be a single SIC-1. That is why the irregular strategy $\mathbf{Y}_{N-1}^{*}$ in Example 2 cannot be an IS-1. Apparently, irregular strategies can have different ranks of their irregularity. Deeper irregularity implies a higher rank of irregular components. As irregular strategies $\mathbf{Y}_{N-1}^{*}$ in PURG may be of higher ranks, Theorem 1 and Theorem 6 must be generalized. 
Theorem 9. Let $\mathscr{U}_{h} \subset\{\overline{1, N-1}\}$ be a subset of those indices within the set $\{\overline{1, N-1}\}$ in PURG, for which inequality

$$
\begin{gathered}
\frac{b_{u_{h}}\left(1-\sum_{w \in \mathscr{A}_{h-1}} a_{w}\right)}{1+\sum_{n=1}^{N-1}\left(b_{n}-a_{n}\right)-\sum_{w \in \mathscr{A}_{h-1}} b_{w}}<a_{u_{h}} \\
\text { for } \mathscr{A}_{h-1}=\bigcup_{q=1}^{h-1} \mathscr{U}_{q} \text { and } u_{h} \in \mathscr{U}_{h}
\end{gathered}
$$

holds successively starting from $h=1$ up to some $h \in \mathbb{N}$ and

$$
\bigcap_{q=1}^{h} \mathscr{U}_{q}=\emptyset \quad \text { for } \quad \mathscr{U}_{q} \neq \emptyset \quad \forall q=\overline{1, h} .
$$

Then the maximal value of the index $h$ is constrained:

$$
h \leqslant N-2 .
$$

Besides,

$$
\mathscr{A}_{h}=\bigcup_{q=1}^{h} \mathscr{U}_{q}
$$

and $\left|\mathscr{A}_{h}\right|$ components of an irregular strategy $\mathbf{Y}_{N-1}^{*}$ are found as

$$
y_{u_{r}}^{*}=a_{u_{r}} \quad \forall u_{r} \in \mathscr{U}_{r} \subset\{\overline{1, N-1}\} \quad \text { by } \quad r=\overline{1, h} .
$$

The rest $N-1-\left|\mathscr{A}_{h}\right|$ components are computed as

$$
y_{k}^{*}=\frac{b_{k}\left(1-\sum_{w \in \mathscr{A}_{h}} a_{w}\right)}{1+\sum_{n=1}^{N-1}\left(b_{n}-a_{n}\right)-\sum_{w \in \mathscr{A}_{h}} b_{w}} \quad \forall k \in\{\overline{1, N-1}\} \backslash \mathscr{A}_{h}
$$

if only

$$
\frac{b_{k}\left(1-\sum_{w \in \mathscr{A}_{h}} a_{w}\right)}{1+\sum_{n=1}^{N-1}\left(b_{n}-a_{n}\right)-\sum_{w \in \mathscr{A}_{h}} b_{w}} \geqslant a_{k} \quad \forall k \in\{\overline{1, N-1}\} \backslash \mathscr{A}_{h} .
$$


Proof. If $h=1$ then $\mathscr{A}_{h-1}=\emptyset$ and it falls directly within conditions of Theorem 6 . By induction for $h \geqslant 2$, the reasoning for (58)-(60) under (55) and (56) is the same as that in Theorem 6 , where $\mathscr{U}$ along the proof is substituted with $\mathscr{A}_{h}$, formula (38) is substituted with

$$
y_{u}^{* *}=\frac{b_{u}\left(1-\sum_{w \in \mathscr{A}_{h-1}} a_{w}\right)}{1+\sum_{n=1}^{N-1}\left(b_{n}-a_{n}\right)-\sum_{w \in \mathscr{A}_{h-1}} b_{w}} \text { for } u \in \mathscr{U} \subset\{\overline{1, N-1}\}
$$

and formula (44) is substituted with

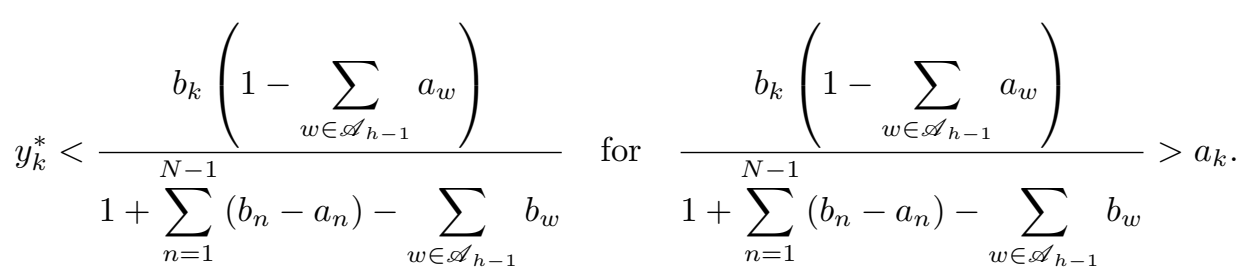

Thus, it remains only to prove (57). The maximal number of non-overlapping nonempty subsets of the set $\{\overline{1, N-1}\}$ is $N-1$ (meaning not every possible combination, but "pieces" of the whole set once broken into them). In this case, they all are singletons. If $h=N-1$ then

$$
\mathscr{A}_{N-1}=\bigcup_{q=1}^{N-1} \mathscr{U}_{q}=\{\overline{1, N-1}\}
$$

and the $k$-th component (59) would be

$$
\begin{aligned}
y_{k}^{*}= & \frac{b_{k}\left(1-\sum_{w \in \mathscr{A}_{N-1}} a_{w}\right)}{1+\sum_{n=1}^{N-1}\left(b_{n}-a_{n}\right)-\sum_{w \in \mathscr{A}_{N-1}} b_{w}} \\
= & \frac{b_{k}\left(1-\sum_{w=1}^{N-1} a_{w}\right)}{1+\sum_{n=1}^{N-1}\left(b_{n}-a_{n}\right)-\sum_{w=1}^{N-1} b_{w}}=\frac{b_{k}\left(1-\sum_{w=1}^{N-1} a_{w}\right)}{1-\sum_{n=1}^{N-1} a_{n}}=b_{k}
\end{aligned}
$$

by $k \in\{\overline{1, N-1}\} \backslash \mathscr{A}_{N-1}=\emptyset$. Hence, the case $h=N-1$ is impossible. If $h=N-2$ then the subset

$$
\mathscr{A}_{N-2}=\bigcup_{q=1}^{N-2} \mathscr{U}_{q}
$$


consists of $N-2$ indices, and thus $k \in\{\overline{1, N-1}\} \backslash \mathscr{A}_{N-2}$ exists as a single index. So, from inequality (60) we have that

$$
\begin{gathered}
\frac{b_{k}\left(1-\sum_{w \in \mathscr{A}_{N-2}} a_{w}\right)}{1+\sum_{n=1}^{N-1}\left(b_{n}-a_{n}\right)-\sum_{w \in \mathscr{A}_{N-2}} b_{w}} \geqslant a_{k}, \\
\frac{b_{k}\left(1-\sum_{w \in \mathscr{A}_{N-2}} a_{w}\right)}{1+b_{k}-\sum_{n=1}^{N-1} a_{n}} \geqslant a_{k}, \\
b_{k}\left(1-\sum_{w \in \mathscr{A}_{N-2}} a_{w}\right) \geqslant a_{k}\left(1+b_{k}-\sum_{n=1}^{N-1} a_{n}\right), \\
b_{k}\left(1-\sum_{w \in \mathscr{A}_{N-2}} a_{w}\right)-a_{k} b_{k} \geqslant a_{k}\left(1-\sum_{n=1}^{N-1} a_{n}\right), \\
b_{k}\left(1-\sum_{w \in \mathscr{A}_{N-2}} a_{w}-a_{k}\right) \geqslant a_{k}\left(1-\sum_{n=1}^{N-1} a_{n}\right), \\
b_{k}\left(1-\sum_{n=1}^{N-1} a_{n}\right) \geqslant a_{k}\left(1-\sum_{n=1}^{N-1} a_{n}\right) .
\end{gathered}
$$

Inequality (63) is true, so that confirms the case $h=N-2$ is possible. Possibility of cases $h<N-2$ is inductively verifiable.

The following definitions generalize ranking the irregularity of the decision-maker's optimal strategy.

Definition 8. The $u_{h}$-th component $y_{u_{h}}^{*}=a_{u_{h}}$ of the second player's optimal strategy $\mathbf{Y}_{N-1}^{*}$ in PURG is called a simple irregular component of the $h$-th rank (SIC- $h$ ) if condition (55) holds, $h=\overline{1, N-2}$. A set of those components (SICs- $h$ ) is called a left strategy subset of the $h$-th rank (LSS- $h$ ).

Definition 9. The $k$-th component (59) of the second player's optimal strategy $\mathbf{Y}_{N-1}^{*}$ in PURG is called a downward-biased irregular component of the $h$-th rank (DBIC- $h$ ), $h=\overline{1, N-2}$. A set of those components (DBICs- $h$ ) is called a biased strategy subset of the $h$-th rank (BSS- $h$ ). 
Definition 10. An irregular optimal strategy $\mathbf{Y}_{N-1}^{*}$ of the second player in PURG is called an irregular strategy of the $h$-th rank (IS- $h$ ) if it includes an LSS- $h$ and does not contain irregular components of ranks higher than $h$.

According to Theorem 9, IS- $h$ always contains SICs- $r \forall r=\overline{1, h}$ and BSS- $h$. Every LSS- $r$ contains at least a one component. IS- $h$ does not contain BSS- $r \forall r=\overline{1, h-1}$. The irregular optimal strategy of the highest rank, which is, literally, IS- $(N-2)$, has a captivating property.

Theorem 10. IS- $(N-2)$ in PURG has a one and only one component, which is greater than the corresponding left endpoint.

Proof. Using (61) from the proof of Theorem 9, we have (58) for $N-2$ indices. Here, inequality (62) holds for a single index $k \in\{\overline{1, N-1}\} \backslash \mathscr{A}_{N-2}$. But inequality (63) always holds strictly. Therefore, inequality (62) holds strictly as well.

Reverting to Example 2, its decision-maker's optimal strategy $\mathbf{Y}_{N-1}^{*}$ is computable now: $\mathscr{U}_{1}=\{2\}$ and a single SIC-1 is $y_{2}^{*}=a_{2}=0.15, \mathscr{U}_{2}=\{3\}$ and a single SIC-2 is $y_{3}^{*}=a_{3}=0.16$. Finally, a single DBIC-2 is

$$
y_{1}^{*}=\frac{b_{1}\left(1-a_{2}-a_{3}\right)}{1+\sum_{n=1}^{3}\left(b_{n}-a_{n}\right)-b_{2}-b_{3}}=\frac{0.5 \cdot(1-0.15-0.16)}{1+0.54-0.2-0.25}=\frac{69}{218}>\frac{1}{10}=a_{1} .
$$

This IS-2 is an instance that supports the assertion of Theorem 10. Inequality (52) within the assertion of Theorem 8 holds as well. Similar to Theorem 9, which generalizes Theorem 6, an inductive generalization to Theorem 8 is important as well.

Theorem 11. Whichever nonempty IS-h in PURG is, $\exists l \in\{\overline{1, N-1}\} \backslash \mathscr{A}_{h}$ such that

$$
\frac{b_{l}\left(1-\sum_{w \in \mathscr{A}_{h}} a_{w}\right)}{1+\sum_{n=1}^{N-1}\left(b_{n}-a_{n}\right)-\sum_{w \in \mathscr{A}_{h}} b_{w}}>a_{l} \quad \text { for } \quad h \in\{\overline{1, N-2}\} .
$$

Proof. Assume that inequality (60) holds with the reverse sign implying that

$$
\frac{b_{k}\left(1-\sum_{w \in \mathscr{A}_{h}} a_{w}\right)}{1+\sum_{n=1}^{N-1}\left(b_{n}-a_{n}\right)-\sum_{w \in \mathscr{A}_{h}} b_{w}} \leqslant a_{k} \quad \forall k \in\{\overline{1, N-1}\} \backslash \mathscr{A}_{h} .
$$

Then, $\mathscr{U}$ is substituted with $\mathscr{A}_{h}$, and we get the same reasoning as that in Theorem 8, finally coming to contradiction (54). The refuted assumption implies that $\exists l \in\{\overline{1, N-1}\} \backslash \mathscr{A}_{h}$ such that (64) holds. 
According to Theorem 11, a case of $y_{k}^{*}=a_{k} \forall k \in\{\overline{1, N-1}\}$ in PURG is impossible. Nevertheless, it is revealed that a potential DBIC- $h$ is always less than a potential DBIC- $(h-1)$.

Theorem 12. DBICs-h and DBICs- $(h-1)$ obey the inequality

$$
\begin{gathered}
b_{k}\left(1-\sum_{w \in \mathscr{A}_{h}} a_{w}\right) \\
1+\sum_{n=1}^{N-1}\left(b_{n}-a_{n}\right)-\sum_{w \in \mathscr{A}_{h}} b_{w} \\
\forall k \in\left\{\frac{b_{k}\left(1-\sum_{z \in \mathscr{A}_{h-1}} a_{z}\right)}{1+\sum_{n=1}^{N-1}\left(b_{n}-a_{n}\right)-\sum_{z \in \mathscr{A}_{h-1}} b_{z}}\right. \\
\backslash \mathscr{A}_{h} \quad \text { and } \forall h=\overline{2, N-2 .}
\end{gathered}
$$

Proof. The difference between the left and right terms in inequality (65) divided by $b_{k}$ is:

$$
\begin{aligned}
((1- & \left.\sum_{w \in \mathscr{A}_{h}} a_{w}\right)\left(1+\sum_{n=1}^{N-1}\left(b_{n}-a_{n}\right)-\sum_{z \in \mathscr{A}_{h-1}} b_{z}\right) \\
& \left.-\left(1-\sum_{z \in \mathscr{A}_{h-1}} a_{z}\right)\left(1+\sum_{n=1}^{N-1}\left(b_{n}-a_{n}\right)-\sum_{w \in \mathscr{A}_{h}} b_{w}\right)\right)\left(1+\sum_{n=1}^{N-1}\left(b_{n}-a_{n}\right)\right. \\
& \left.-\sum_{w \in \mathscr{A}_{h}} b_{w}\right)^{-1}\left(1+\sum_{n=1}^{N-1}\left(b_{n}-a_{n}\right)-\sum_{z \in \mathscr{A}_{h-1}} b_{z}\right)^{-1}
\end{aligned}
$$

Using that $\mathscr{A}_{h}=\mathscr{A}_{h-1} \cup \mathscr{U}_{h}$ and

$$
\begin{aligned}
\sum_{w \in \mathscr{A}_{h}} a_{w} & =\sum_{z \in \mathscr{A}_{h-1}} a_{z}+\sum_{l \in \mathscr{U}_{h}} a_{l}, \\
\sum_{w \in \mathscr{A}_{h}} b_{w} & =\sum_{z \in \mathscr{A}_{h-1}} b_{z}+\sum_{l \in \mathscr{U}_{h}} b_{l},
\end{aligned}
$$

the expanded numerator in (66) is:

$$
\begin{aligned}
1+\sum_{n=1}^{N-1}\left(b_{n}-a_{n}\right)-\sum_{z \in \mathscr{A}_{h-1}} b_{z}-\sum_{w \in \mathscr{A}_{h}} a_{w} \\
-\sum_{w \in \mathscr{A}_{h}} a_{w} \sum_{n=1}^{N-1}\left(b_{n}-a_{n}\right)+\sum_{w \in \mathscr{A}_{h}} a_{w} \sum_{z \in \mathscr{A}_{h-1}} b_{z} \\
-1-\sum_{n=1}^{N-1}\left(b_{n}-a_{n}\right)+\sum_{w \in \mathscr{A}_{h}} b_{w}+\sum_{z \in \mathscr{A}_{h-1}} a_{z}
\end{aligned}
$$




$$
\begin{aligned}
& +\sum_{z \in \mathscr{A}_{h-1}} a_{z} \sum_{n=1}^{N-1}\left(b_{n}-a_{n}\right)-\sum_{z \in \mathscr{A}_{h-1}} a_{z} \sum_{w \in \mathscr{A}_{h}} b_{w} \\
& =-\sum_{l \in \mathscr{U}_{h}} a_{l}-\sum_{l \in \mathscr{U}_{h}} a_{l} \sum_{n=1}^{N-1}\left(b_{n}-a_{n}\right) \\
& +\sum_{w \in \mathscr{A}_{h}} a_{w} \sum_{z \in \mathscr{A}_{h-1}} b_{z}+\sum_{l \in \mathscr{U}_{h}} b_{l}-\sum_{z \in \mathscr{A}_{h-1}} a_{z} \sum_{w \in \mathscr{A}_{h}} b_{w} \\
& =-\sum_{l \in \mathscr{U}_{h}} a_{l}\left(1+\sum_{n=1}^{N-1}\left(b_{n}-a_{n}\right)\right)+\left(\sum_{z \in \mathscr{A}_{h}-1} a_{z}+\sum_{l \in \mathscr{U}_{h}} a_{l}\right) \sum_{z \in \mathscr{A}_{h-1}} b_{z} \\
& +\sum_{l \in \mathscr{U}_{h}} b_{l}-\sum_{z \in \mathscr{A}_{h-1}} a_{z}\left(\sum_{z \in \mathscr{A}_{h-1}} b_{z}+\sum_{l \in \mathscr{U}_{h}} b_{l}\right) \\
& =-\sum_{l \in \mathscr{U}_{h}} a_{l}\left(1+\sum_{n=1}^{N-1}\left(b_{n}-a_{n}\right)\right) \\
& +\sum_{l \in \mathscr{U}_{h}} a_{l} \sum_{z \in \mathscr{A}_{h-1}} b_{z}+\sum_{l \in \mathscr{U}_{h}} b_{l}-\sum_{z \in \mathscr{A}_{h-1}} a_{z} \sum_{l \in \mathscr{U}_{h}} b_{l} \\
& =-\sum_{l \in \mathscr{U}_{h}} a_{l}\left(1+\sum_{n=1}^{N-1}\left(b_{n}-a_{n}\right)-\sum_{z \in \mathscr{A}_{h-1}} b_{z}\right) \\
& +\sum_{l \in \mathscr{U}_{h}} b_{l}\left(1-\sum_{z \in \mathscr{A}_{h-1}} a_{z}\right) \text {. }
\end{aligned}
$$

Summing up both sides of (55), we get

$$
\begin{gathered}
\frac{\sum_{u_{h} \in \mathscr{U}_{h}} b_{u_{h}}\left(1-\sum_{w \in \mathscr{A}_{h-1}} a_{w}\right)}{1+\sum_{n=1}^{N-1}\left(b_{n}-a_{n}\right)-\sum_{w \in \mathscr{A}_{h-1}} b_{w} a_{u_{h} \in \mathscr{U}_{h}} a_{u_{h}},} \\
\sum_{u_{h} \in \mathscr{U}_{h}} b_{u_{h}}\left(1-\sum_{w \in \mathscr{A}_{h-1}} a_{w}\right)-\sum_{u_{h} \in \mathscr{U}_{h}} a_{u_{h}}\left(1+\sum_{n=1}^{N-1}\left(b_{n}-a_{n}\right)-\sum_{w \in \mathscr{A}_{h-1}} b_{w}\right)<0,
\end{gathered}
$$

whence the last term of (67) is negative. Thus, difference (66) is negative, so inequality (65) holds. 
Henceforward, strategy $\mathbf{Y}_{N-1}^{*}$ in PURG is either regular or irregular. Whichever strategy $\mathbf{Y}_{N-1}^{*}$ is, its $k$-th component is (29) following Theorem 7 and Theorem 12.

\section{Application}

Branches, where PURG and the strategy $\mathbf{Y}_{N-1}^{*}$ may be applied, relate to multiparametric systems whose statistics either are very poor or include a high volatility. These systems must consist of $N$ objects, over which some action is accomplished. Delivering capacities, loading/charging up to uncertain levels, time windowing/scheduling are the most attractable examples of the action $[44,45,19,40$, 10]. Volumes or periods thereby are either non-predictable or their adjustment takes significant time/resources/energy.

An instance of the PURG's application is an incipient consumer service, where amounts of demands at the start are given as intervals, although the grand total is fixed. The $N$-th consumer is a fictional one, whose "demand" is an unclaimed amount. This amount is usually delivered backward or utilized. If all consumers are equal (without priorities), the PURG-based model with (30)-(32) is acceptable.

If the amounts are areas to be appropriately divided, then $q=2$. Cases with $q=2$ relate mostly to areas/squares when intervals are given in feet, yards, miles, etc. If the amounts are measured in cubature (say, for gallonage, barrels, or cubic meters), then $q=3$. Cases with $q=3$ relate to volumes when intervals are given similarly. The power $q$, although not influencing on the strategy $\mathbf{Y}_{N-1}^{*}$, stands for the factual results and the point estimation aftermaths.

PURG can be used in preparing data for interval analysis, without mapping intervals into points. The data preparation purports refinement of intervals. If an irregularity is revealed then the intervals may be considered inappropriate for operating over them. Thus, the presence of irregular components in $\mathbf{Y}_{N-1}^{*}$ is a criterion for preventing improper interval operations. Only regular strategy $\mathbf{Y}_{N-1}^{*}$ or $\mathbf{Y}_{N-1}^{*}$ with just DBICs-1 and a few SICs-1 might admit operations over the corresponding $N-1$ intervals.

Example 3. Let $N=5, a_{1}=0.175, b_{1}=0.225, a_{2}=0.2, b_{2}=0.225, a_{3}=0.2$, $b_{3}=0.25, a_{4}=0.15, b_{4}=0.275$. Here, first of all,

$$
\begin{aligned}
\sum_{n=1}^{4}\left(b_{n}-a_{n}\right)= & 0.225-0.175+0.225-0.2+0.25-0.2+0.275-0.15=0.25, \\
& \frac{b_{1}}{1+\sum_{n=1}^{4}\left(b_{n}-a_{n}\right)}=\frac{0.225}{1+0.25}=\frac{9}{50}>\frac{7}{40}=a_{1}, \\
& \frac{b_{2}}{1+\sum_{n=1}^{4}\left(b_{n}-a_{n}\right)}=\frac{0.225}{1+0.25}=\frac{9}{50}<\frac{1}{5}=a_{2},
\end{aligned}
$$




$$
\begin{gathered}
\frac{b_{3}}{1+\sum_{n=1}^{4}\left(b_{n}-a_{n}\right)}=\frac{0.25}{1+0.25}=\frac{1}{5}=a_{3}, \\
\frac{b_{4}}{1+\sum_{n=1}^{4}\left(b_{n}-a_{n}\right)}=\frac{0.275}{1+0.25}=\frac{11}{50}>\frac{3}{20}=a_{4},
\end{gathered}
$$

whence $\mathscr{U}_{1}=\{2\}$ and $y_{2}^{*}=a_{2}=0.2$. Then

$$
\begin{aligned}
& \frac{b_{1}\left(1-a_{2}\right)}{1+\sum_{n=1}^{4}\left(b_{n}-a_{n}\right)-b_{2}}=\frac{0.225 \cdot(1-0.2)}{1+0.25-0.225}=\frac{36}{205}>\frac{7}{40}=a_{1}, \\
& \frac{b_{3}\left(1-a_{2}\right)}{1+\sum_{n=1}^{4}\left(b_{n}-a_{n}\right)-b_{2}}=\frac{0.25 \cdot(1-0.2)}{1+0.25-0.225}=\frac{8}{41}<\frac{1}{5}=a_{3}, \\
& \frac{b_{4}\left(1-a_{2}\right)}{1+\sum_{n=1}^{4}\left(b_{n}-a_{n}\right)-b_{2}}=\frac{0.275 \cdot(1-0.2)}{1+0.25-0.225}=\frac{44}{205}>\frac{3}{20}=a_{4},
\end{aligned}
$$

whence $\mathscr{U}_{2}=\{3\}$ and $y_{3}^{*}=a_{3}=0.2$. Further,

$$
\begin{aligned}
& \frac{b_{1}\left(1-a_{2}-a_{3}\right)}{1+\sum_{n=1}^{4}\left(b_{n}-a_{n}\right)-b_{2}-b_{3}}=\frac{0.225 \cdot(1-0.2-0.2)}{1+0.25-0.225-0.25}=\frac{27}{155}<\frac{7}{40}=a_{1}, \\
& \frac{b_{4}\left(1-a_{2}-a_{3}\right)}{1+\sum_{n=1}^{4}\left(b_{n}-a_{n}\right)-b_{2}-b_{3}}=\frac{0.275 \cdot(1-0.2-0.2)}{1+0.25-0.225-0.25}=\frac{33}{155}>\frac{3}{20}=a_{4},
\end{aligned}
$$

whence $\mathscr{U}_{3}=\{1\}$ and $y_{1}^{*}=a_{1}=0.175$. Finally, according to Theorem 10 ,

$$
\begin{aligned}
y_{4}^{*} & =\frac{b_{4}\left(1-a_{2}-a_{3}-a_{1}\right)}{1+\sum_{n=1}^{4}\left(b_{n}-a_{n}\right)-b_{2}-b_{3}-b_{1}} \\
& =\frac{0.275 \cdot(1-0.2-0.2-0.175)}{1+0.25-0.225-0.25-0.225}=\frac{17}{80}>\frac{3}{20}=a_{4} .
\end{aligned}
$$


The found IS-3 is obviously poor. But after corrections of the first three intervals to $[0.165 ; 0.225],[0.17 ; 0.235],[0.19 ; 0.26],[0.15 ; 0.275]$ a totally regular strategy is obtained. The intervals herein are made wider for $20 \%, 160 \%$, and $40 \%$, respectively (the second interval was initially too short).

It is quite clear that the cost $v_{\text {opt }}$ of mapping interval uncertainties into point estimates by the minimax approach is always lesser than the cost by any other point estimation:

$$
\begin{aligned}
v_{\mathrm{opt}}=\max \left\{\left\{\left(\frac{b_{k}}{y_{k}^{*}}\right)^{q}\right\}_{k=1}^{N-1},\left(\frac{1-\sum_{i=1}^{N-1} a_{i}}{1-\sum_{j=1}^{N-1} y_{j}^{*}}\right)^{q}\right\} \\
\leqslant \max \left\{\left\{\left(\frac{b_{k}}{y_{k}^{\langle 0\rangle}}\right)^{q}\right\}_{k=1}^{N-1},\left(\frac{1-\sum_{i=1}^{N-1} a_{i}}{1-\sum_{j=1}^{N-1} y_{j}^{\langle 0\rangle}}\right)^{q}\right\}=v^{\langle 0\rangle},
\end{aligned}
$$

where $\left\{y_{m}^{\langle 0\rangle}\right\}_{m=1}^{N-1}$ are point estimates the first $N-1$ intervals, and

$$
y_{N}^{\langle 0\rangle}=1-\sum_{m=1}^{N-1} y_{m}^{\langle 0\rangle} \text {. }
$$

Point estimates $\left\{y_{m}^{\langle 0\rangle}\right\}_{m=1}^{N-1}$ can be obtained, for example, by simply finding an arithmetic/geometric mean of left and right endpoints for every interval. The numerical analysis, based on randomly generated positive intervals $\left\{\left[a_{k} ; b_{k}\right]\right\}_{k=1}^{N-1}$ by (3), shows that non-minimax cost $v^{\langle 0\rangle}$ for both arithmetic and geometric mean approaches is far greater that that of minimax (Figure 2). The cost of choosing the point estimates as values of random variables is obviously the worst.

The average costs of the point estimation inevitably grow as $N$ increases. The minimax cost growth is significantly slower (Figure 3 ). Moreover, it looks like it has an asymptote, which is less than 1.3 for a reasonably wide sequence of $N$. The growths of the cost for both arithmetic and geometric mean approaches along with the random point choice do not seem to have such asymptotes. Nonetheless, the worst minimax cost $v_{\text {opt }}>1.4$ (before averaging in Figure 3).

The advantage of the minimax approach reflected in Figure 3 strengthens as the number of intervals to be mapped into point estimates increases. Despite the minimax cost expectedly grows, prevention of the worst event is the main goal. This is especially important for branches where consequences of mistaken decisions will cost far much more than $v_{\text {opt }}$ by (68), e.g., power management systems planning [4, 32], allocation of resources for sustainability $[24,19,25]$, nuclear data processing (1) and design of reactor in-core monitoring systems [16], reliable flight trajectories [28], cybersecurity $[30,25]$. 


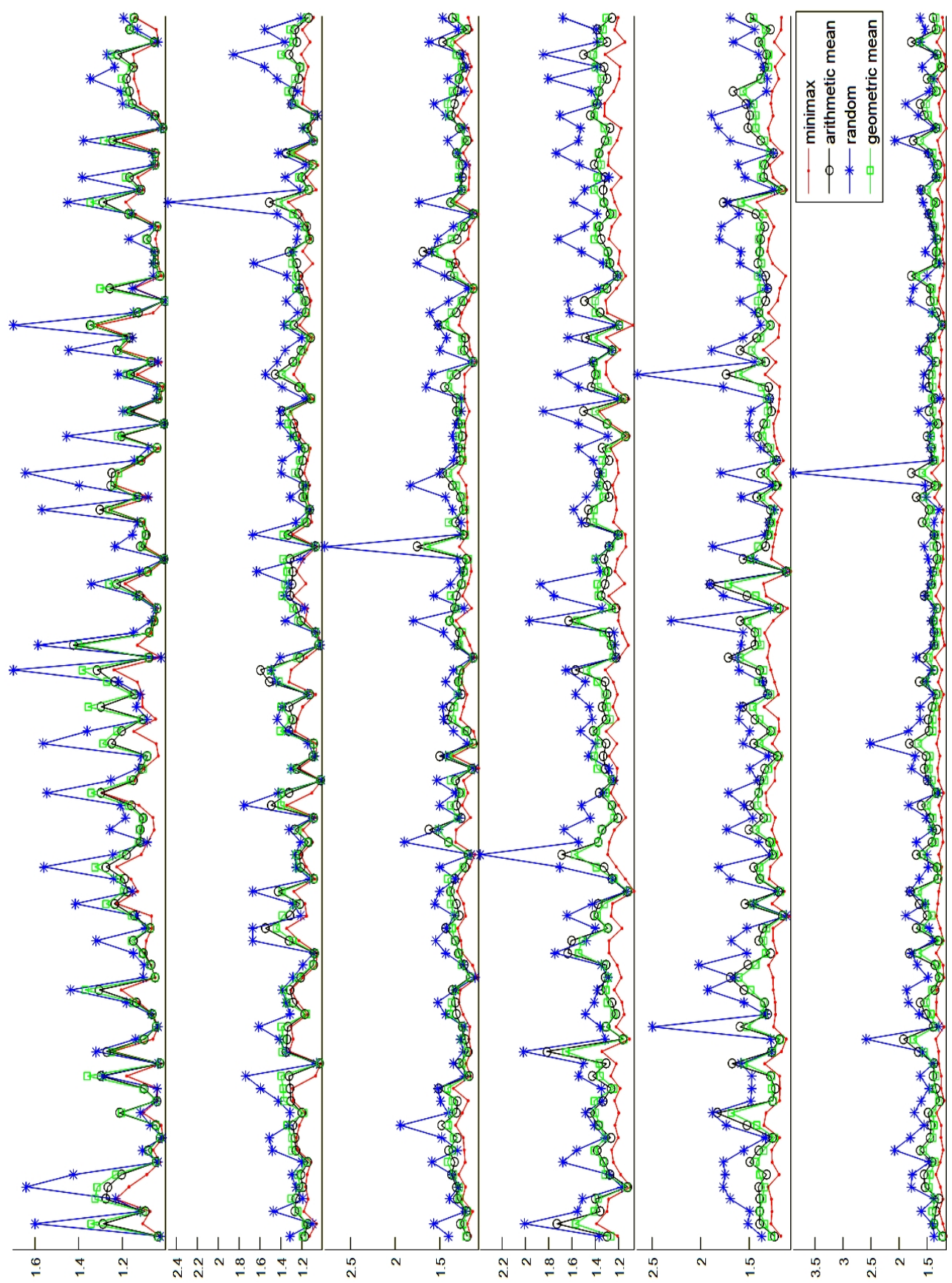

Figure 2: Costs of the point estimation (for $q=1$ ) for six series of 100 sets of intervals by increasing $N$ from 2 through 7 (moving from the top to bottom), where the maximal ratio of the right and left endpoints is not greater than 2 


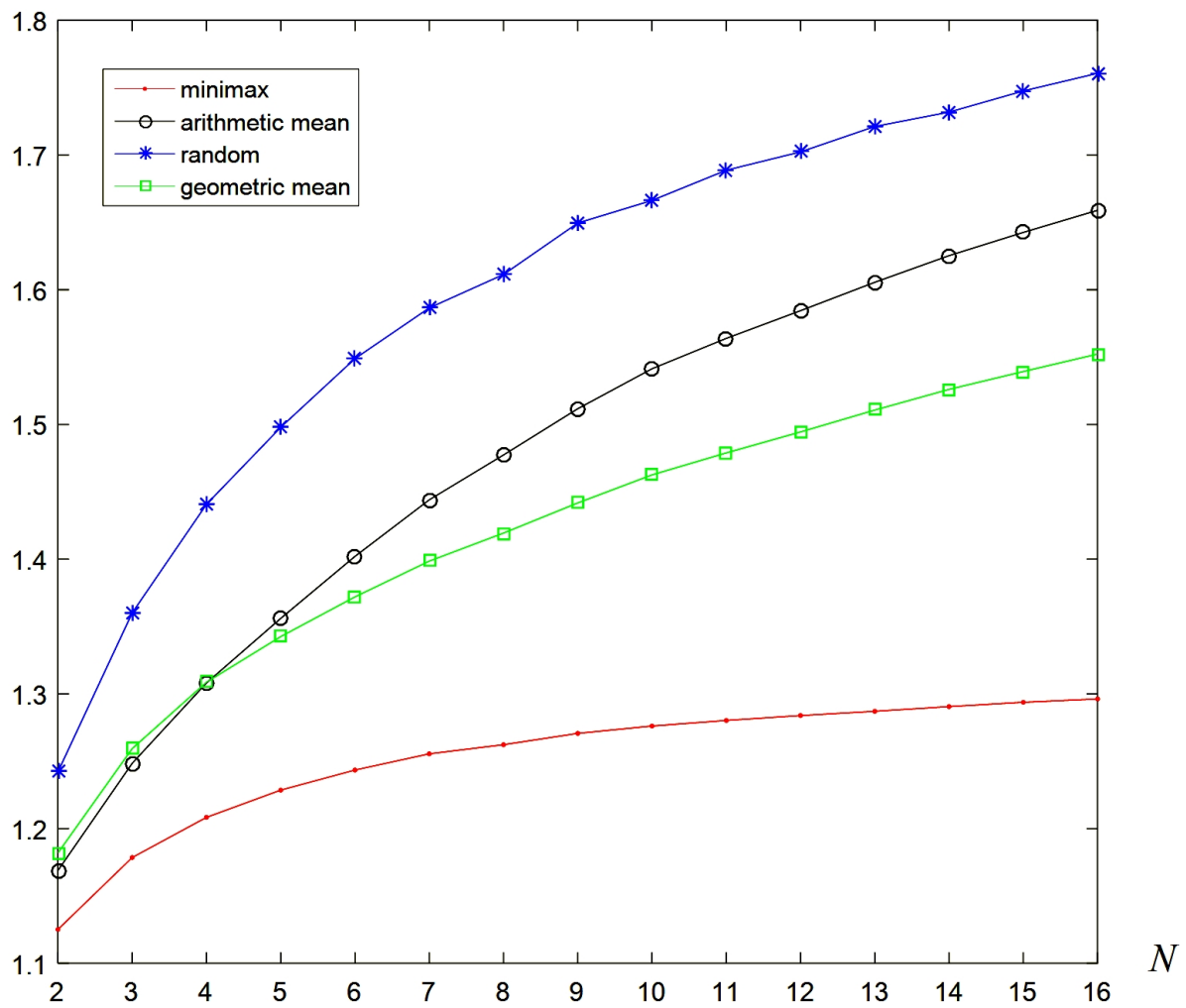

Figure 3: The average costs of the point estimation (for $q=1$ ) for 15 series of 5000 sets of intervals, where the maximal ratio of the right and left endpoints is not greater than 2

\section{Discussion}

An apparent demerit of the PURG-based model is that the decision-maker's optimal strategy $\mathbf{Y}_{N-1}^{*}$ becomes inefficient as statistical data are accumulated. When statistical data are still insufficient for Bayesian decisions, the minimax strategy can be softened with expectations $[27,51]$. A suitable moment to stop using the minimax approach along with the PURG-based model is when the respective probability density functions can be elicited from the accumulated statistics. However, strategy $\mathbf{Y}_{N-1}^{*}$ appears efficient when external conditions change frequently/quickly (i.e., are highly volatile) regardless of whether statistical data are sufficient or not.

Figure 4 shows interrelation of the proven theorems. Simpler cases are enveloped in more general cases. Theorem 4 describes an ideal version of PURG. It is naively believed that approximating to such PURG will give a totally regular strategy for the decision-maker. 


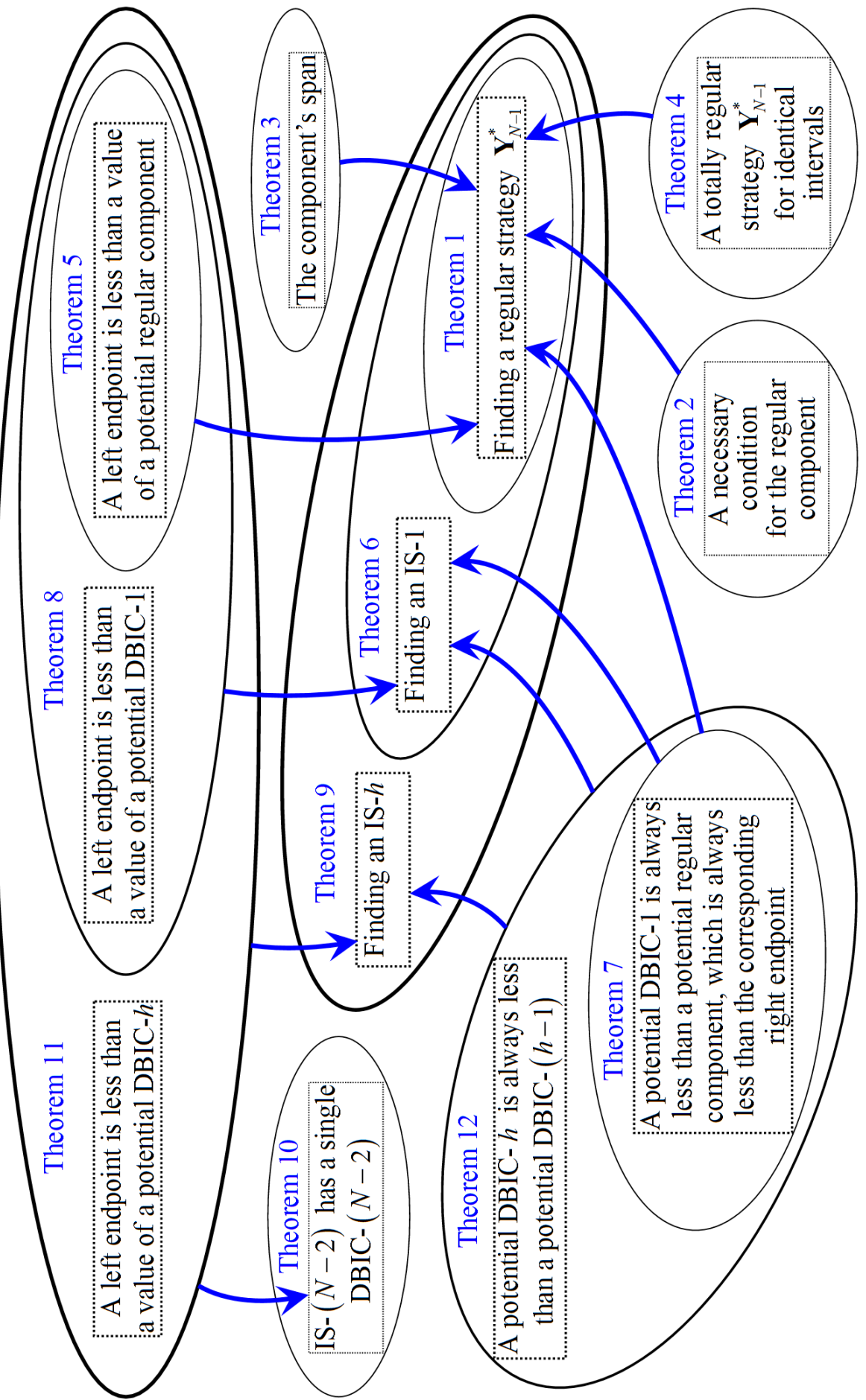

Figure 4: Assertions and purposes of the proven theorems 
Normalization to 1 allows considering a fluent grand total, but the endpoints of $N-1$ intervals must be fixed. This means that the portion of a particular interval should be always fixed. This is the fundamental step while dealing with real interval data.

Simple realizability in practice is a merit of the PURG-based model. Another one is a non-sophisticated routine of building PURG and finding the minimax strategy. This routine is of two stages. Firstly, grand total and boundaries of objects' portions are normalized. Secondly, components $\left\{y_{k}^{*}\right\}_{k=1}^{N-1}$ are computed, whichever formulae are used. Moreover, the solution is independent of $q$. Such independency imparts some universality to PURG.

Strategy $\mathbf{Y}_{N-1}^{*}$ is always single, there are no PURGs with a continuum of such strategies. This saves from solving a subsequent decision making problem that lies in selecting a single unique strategy. Additionally, the minimax irregular strategy is ranked, that facilitate in comparing different intervals given before the point estimation. Intervals which are mapped into simple irregular components of higher ranks are less reliable.

Notwithstanding the intervals and IS-3 in Example 3, a series of numerical tests confirms that cases of deeply irregular components (high ranks of irregularity) are unlikely. Unlikelihood strengthens when endpoints of the same type (left or right) are close by value, especially if they are identical. A confluent case is PURG on hypercube (30) granting a totally regular strategy with components (32).

\section{Conclusion}

The PURG-based model, including formulae for $\left\{y_{k}^{*}\right\}_{k=1}^{N-1}$ and (11), is a contribution to the field of non-statistical interval uncertainty reduction. This is a model of maximal disbalance between a real unknown amount and a guessed amount. These amounts are interpreted as aftermaths of the point estimation. This model grants a pure strategy $\mathbf{Y}_{N-1}^{*}$ whose components are the most appropriate point estimates. The appropriateness is founded on the minimax principle. This is a subtle optimization model addressing minimum information processes. The approach will work efficiently only by when no information is available but those interval estimates. Such a situation is common in processing raw data of difficult measurements and measurements with jeopardy (for instance, concentrations of river/air/ground pollutants, radiation control, etc.), where statistics are too small to estimate, e.g., a probability distribution and find point estimates of its main characteristics (expectance and variance). The minimax approach is applicable for cases just like those mentioned above, when the risk of a biased decision should be as minimal as possible until additional information becomes available.

Main benefits of PURG and $\mathbf{Y}_{N-1}^{*}$ are simplicity and acceptability of any time period for practical realization. This is because we do not have expected values, but only ready-to-go values. In other words, decisions based on minimax strategy $\mathbf{Y}_{N-1}^{*}$ are implemented on-the-fly, whichever changes of initial intervals are, and the result of such an implementation comes instantly. If something new emerges frequently 
(resulting in frequently "floating" intervals), the minimax reaction by strategy $\mathbf{Y}_{N-1}^{*}$ is immediate owing to its components are re-computed easily.

Apart from the point estimation, the PURG-based model contributes to the decision making theory by deciding on appropriateness of the initial interval estimations grouped as (2) by (3). Irregularities in the decision-maker's optimal strategy $\mathbf{Y}_{N-1}^{*}$ may serve as an evidence of incorrectly setting the corresponding intervals' endpoints. Furthermore, higher ranks of irregularity might reject the corresponding intervals and send them for correction before starting interval calculus. More specifically, a totally regular strategy $\mathbf{Y}_{N-1}^{*}$ satisfying condition (28) is the best case for mapping partial interval uncertainties into point estimates. A regular strategy $\mathbf{Y}_{N-1}^{*}$ found by (12) but failing with (28) produces a suspicion of that not all the intervals are evaluated correctly. An irregular strategy of the first rank (IS-1) found by (34)-(37) is a feature of poorly evaluated intervals. Then, correction of the intervals corresponding to LSS-1 (a set of SICs-1) is an option. Deeper irregularity implying a higher rank of irregular components directs that option to compulsorily correcting the intervals which correspond to left strategy subsets of all the ranks reached. Therefore, LSS-2 and left strategy subsets of higher ranks play a role of indicators to which intervals should be necessarily corrected.

\section{References}

[1] E. Alhassan, H. Sjöstrand, P. Helgesson, M. Österlund, S. Pomp, A.J. Koning, D. Rochman, On the use of integral experiments for uncertainty reduction of reactor macroscopic parameters within the TMC methodology, Progress in Nuclear Energy 88 (2016) 43-52.

[2] M.H. Bazerman, D.A. Moore, Judgment in Managerial Decision Making (8th ed.), Wiley, River Street, Hoboken, NJ, 2013.

[3] J.O. Berger, Minimax analysis, in: Statistical Decision Theory and Bayesian Analysis, J.O. Berger (ed.), Springer, New York, NY, 1985, 308-387.

[4] C. Dong, G.H. Huang, Y.P. Cai, Y. Xu, An interval-parameter minimax regret programming approach for power management systems planning under uncertainty, Applied Energy 88 (8) (2011) 2835-2845.

[5] J.P.C. Driessen, H. Peng, G.J. van Houtum, Maintenance optimization under non-constant probabilities of imperfect inspections, Reliability Engineering \& System Safety 165 (2017) 115-123.

[6] Y.C. Eldar, Minimax estimation of deterministic parameters in linear models with a random model matrix, IEEE Transactions on Signal Processing 54 (2) (2006) 601-612.

[7] R. Festa, Bayesian point estimation, verisimilitude, and immodesty, in: Optimum Inductive Methods, R. Festa (ed.), Springer, Dordrecht, 1993, 38-47. 
[8] C. Fu, X. Ren, Y. Yang, W. Qin, Dynamic response analysis of an overhung rotor with interval uncertainties, Nonlinear Dynamics 89 (3) (2017) 2115-2124.

[9] E. Ghashim, É. Marchand, W.E. Strawderman, On a better lower bound for the frequentist probability of coverage of Bayesian credible intervals in restricted parameter spaces, Statistical Methodology 31 (2016) 43-57.

[10] M. González, C. Minuesa, I. del Puerto, Maximum likelihood estimation and expectation-maximization algorithm for controlled branching processes, Computational Statistics \& Data Analysis 93 (2016) 209-227.

[11] G.C. Goodwin, R.L. Payne, Dynamic System Identification: Experiment Design and Data Analysis, Academic Press, New York, NY, 1977.

[12] P. Guo, G.H. Huang, Y.P. Li, Inexact fuzzy-stochastic programming for water resources management under multiple uncertainties, Environmental Modeling \& Assessment 15 (2) (2010) 111-124.

[13] P. Guo, H. Tanaka, Decision making with interval probabilities, European Journal of Operational Research 203 (2) (2010) 444-454.

[14] M.A. Howe, B. Rustem, M.J.P. Selby, Multi-period minimax hedging strategies, European Journal of Operational Research 93 (1) (1996) 185-204.

[15] A. Jablonski, T. Barszcz, M. Bielecka, P. Breuhaus, Modeling of probability distribution functions for automatic threshold calculation in condition monitoring systems, Measurement 46 (1) (2013) 727-738.

[16] Y. Kobayashi, K. Okabe, S. Kondo, Y. Togo, Application of minimax principle to design of reactor in-core monitoring system, Journal of Nuclear Science and Technology 10 (12) (1973) 731-738.

[17] E.L. Lehmann, G. Casella, Theory of Point Estimation (2nd ed.), Springer, New York, NY, 1998.

[18] M. Leonelli, J.Q. Smith, Directed expected utility networks, Decision Analysis 14 (2) (2017) 108-125.

[19] Y.P. Li, G.H. Huang, S.L. Nie, A robust interval-based minimax-regret analysis approach for the identification of optimal water-resources-allocation strategies under uncertainty, Resources, Conservation and Recycling 54 (2) (2009) 86-96.

[20] J. Liebowitz, The Handbook of Applied Expert Systems, CRC Press, Boca Raton, FL, 1997.

[21] P. Liu, F. Jin, X. Zhang, Y. Su, M. Wang, Research on the multi-attribute decision-making under risk with interval probability based on prospect theory and the uncertain linguistic variables, Knowledge-Based Systems 24 (4) (2011) $554-561$. 
[22] Y.K. Liu, The completion of a fuzzy measure and its applications, Fuzzy Sets and Systems 123 (2) (2001) 137-145.

[23] W.A. Lodwick, K.D. Jamison, Interval-valued probability in the analysis of problems containing a mixture of possibilistic, probabilistic, and interval uncertainty, Fuzzy Sets and Systems 159 (21) (2008) 2845-2858.

[24] H. Luss, Minimax resource allocation problems: Optimization and parametric analysis, European Journal of Operational Research 60 (1) (1992) 76-86.

[25] W. Ma, K. McAreavey, W. Liu, X. Luo, Acceptable costs of minimax regret equilibrium: a solution to security games with surveillance-driven probabilistic information, Expert Systems with Applications 108 (2018) 206-222.

[26] M.J. Machina, W.K. Viscusi, Handbook of the Economics of Risk and Uncertainty. Volume 1, North-Holland, Oxford, UK, 2014.

[27] C.A. Martínez, K. Khare, M.A. Elzo, On the Bayesness, minimaxity and admissibility of point estimators of allelic frequencies, Journal of Theoretical Biology 383 (2015) 106-115.

[28] A. Miele, T. Wang, C.Y. Tzeng, W.W. Melvin, Transformation techniques for minimax optimal control problems and their application to optimal flight trajectories in a windshear: optimal abort landing trajectories, IFAC Proceedings Volumes 20 (5/8) (1987) 131-150.

[29] S. Mishra, A. Datta-Gupta, Multivariate data analysis, in: Applied Statistical Modeling and Data Analytics, S. Mishra, A. Datta-Gupta (eds.), Elsevier, 2018, 97-118.

[30] J. Moon, T. Başar, Minimax control over unreliable communication channels, Automatica 59 (2015) 182-193.

[31] D.E. Morris, J.E. Oakley, J.A. Crowe, A web-based tool for eliciting probability distributions from experts, Environmental Modelling \& Software 52 (2014) 1-4.

[32] C. Ning, F. You, Adaptive robust optimization with minimax regret criterion: Multiobjective optimization framework and computational algorithm for planning and scheduling under uncertainty, Computers \& Chemical Engineering 108 (2018) 425-447.

[33] N. Nisan, T. Roughgarden, É. Tardos, V.V. Vazirani, Algorithmic Game Theory, Cambridge University Press, Cambridge, UK, 2007.

[34] L. Pan, D.N. Politis, Bootstrap prediction intervals for Markov processes, Computational Statistics \& Data Analysis 100 (2016) 467-494.

[35] G. Parmigiani, L. Inoue, Decision Theory: Principles and Approaches, Wiley, Chichester, UK, 2009. 
[36] M.M. Rajabi, B. Ataie-Ashtiani, Efficient fuzzy Bayesian inference algorithms for incorporating expert knowledge in parameter estimation, Journal of Hydrology 536 (2016) 255-272.

[37] R.T. Rockafellar, Convex Analysis, Princeton University Press, Princeton, NJ, 1970.

[38] V.V. Romanuke, A generalized model of removing $N$ partial indeterminancies of the probabilistic type as a continuous antagonistic game on $(2 N-2)$-dimensional parallelepiped by maximal disbalance minimization, Herald of Khmelnytskyi National University. Technical Sciences 3 (2011) 45-60.

[39] V.V. Romanuke, Convergence and estimation of the process of computer implementation of the optimality principle in matrix games with apparent play horizon, Journal of Automation and Information Sciences 45 (10) (2013) 49-56.

[40] V.V. Romanuke, Designer's optimal decisions to fit cross-section squares of the supports of a construction platform in overestimations of uncertainties in the generalized model, Cybernetics and Systems Analysis 50 (3) (2014) 426-438.

[41] V.V. Romanuke, Interval uncertainty reduction via division-by-2 dichotomization based on expert estimations for short-termed observations, Journal of Uncertain Systems 12 (1) (2018) 3-21.

[42] S. Tesfamariam, K. Goda, Handbook of Seismic Risk Analysis and Management of Civil Infrastructure Systems, Woodhead Publishing, Cambridge, UK, 2013.

[43] A.D. Torshizi, M.H.F. Zarandi, Hierarchical collapsing method for direct defuzzification of general type-2 fuzzy sets, Information Sciences 277 (2014) 842-861.

[44] N.N. Vorob'yov, Game Theory Fundamentals. Noncooperative Games, Nauka, Moscow, 1984 (in Russian).

[45] N.N. Vorob'yov, Game Theory for Economists-Cyberneticists, Nauka, Moscow, 1985 (in Russian).

[46] R.E. Walpole, R.H. Myers, S.L. Myers, K. Ye, Probability \&3 Statistics for Engineers \& Scientists (9th ed.), Prentice Hall, Boston, MA, 2012.

[47] É. Walter, L. Pronzato, Identification of Parametric Models from Experimental Data, Springer, New York, NY, 1997.

[48] M. Xia, C.S. Cai, F. Pan, Y. Yu, Estimation of extreme structural response distributions for mean recurrence intervals based on short-term monitoring, Engineering Structures 126 (2016) 121-132.

[49] R.C. Yadava, P.K. Rai, Analyzing variety of birth intervals: A stochastic approach, in: Handbook of Statistics. Volume 40, A.S.R.S. Rao, C.R. Rao (eds.), Elsevier, 2019, 195-283. 
[50] Y. Zhou, N. Fenton, M. Neil, Bayesian network approach to multinomial parameter learning using data and expert judgments, International Journal of Approximate Reasoning 55 (5) (2014) 1252-1268.

[51] S. Zinodiny, S. Rezaei, S. Nadarajah, Bayes minimax estimation of the multivariate normal mean vector under balanced loss function, Statistics \& Probability Letters 93 (2014) 96-101.

\section{DOI: $10.7862 /$ rf.2019.10}

\section{Vadim Romanuke}

email: v.romanuke@amw.gdynia.pl

ORCID: 0000-0003-3543-3087

Faculty of Navigation and Naval Weapons

Polish Naval Academy

Gdynia

POLAND 